\title{
Disentangling new physics effects on nonresonant Higgs boson pair production from gluon fusion
}

\author{
Kingman Cheung, ${ }^{1,2,3, *}$ Adil Jueid $\odot,{ }^{1, \dagger}$ Chih-Ting Lu๑ ${ }^{4, *}$ Jeonghyeon Song $\oplus^{1,8}$ and Yeo Woong Yoon ${ }^{5, \|}$ \\ ${ }^{1}$ Department of Physics, Konkuk University, 120 Neungdong-ro, Gwangjin-gu, \\ Seoul 05029, Republic of Korea \\ ${ }^{2}$ Physics Division, National Center for Theoretical Sciences, Hsinchu, Taiwan \\ ${ }^{3}$ Department of Physics, National Tsing Hwa University, Hsinchu 300, Taiwan \\ ${ }^{4}$ School of Physics, KIAS, 85 Hoegiro, Dongdaemun-gu, Seoul 02455, Republic of Korea \\ ${ }^{5}$ School of Liberal Arts, Seoul-Tech, Seoul 139-743, Republic of Korea
}

(Received 8 May 2020; accepted 21 December 2020; published 15 January 2021)

\begin{abstract}
We study the kinematic characteristics of the loop-induced effects on nonresonant Higgs pair production via gluon fusion in a type-II two Higgs doublet model with heavy vectorlike quarks. First, we performed a comprehensive analysis of the model phenomenology, suggesting an ansatz to satisfy theoretical and experimental constraints. Contrary to the usual expectation, the loop-induced effects can highly enhance the di-Higgs rate if the Higgs sector is extended into the wrong-sign limit $\left(\kappa_{t}=-\kappa_{b}=1\right)$ : the total cross section can be about three times as large as the SM expectation. As anomalous Higgs trilinear self-coupling of $\lambda_{h h h} / \lambda_{h h h}^{\mathrm{SM}}=-0.5,5.5$ similarly enhances the di-Higgs rate, we made a comparative study focusing on the kinematic distributions. The threshold effects from the vectorlike quarks generate the correlated bumps in the distributions of the invariant mass of the Higgs boson pair and the transverse momentum of a Higgs boson, located at $M_{h h} \simeq 2 M_{\mathrm{VLQ}}$ and $p_{T}^{h} \simeq M_{\mathrm{VLQ}}$. Each bump has a long tail stretching toward the high region. The anomalous $\lambda_{\text {hhh }}$ cases shift the distributions into low regions. In addition, we show the kinematic difference between the loop-induced effects and an $s$-channel wide resonance. Therefore, the kinematic regimes with high $M_{h h}$ and high $p_{T}^{h}$ would have high potential to probe the loop-induced effects on the di-Higgs process, which is shown through full HL-LHC simulations in the $b \bar{b} b \bar{b}$ and $b \bar{b} \gamma \gamma$ final states.
\end{abstract}

DOI: 10.1103/PhysRevD.103.015019

\section{INTRODUCTION}

In particle physics, a great step forward in knowledge or model building has always been realized by the observation of a new interaction vertex. The discovery of a Higgs boson at the LHC by the ATLAS and CMS collaborations [1,2] was based on the measurement of the couplings of a new scalar boson to vector bosons and the third generation fermions. Even though all of the experimental results conform to the phenomenology of the standard model (SM) Higgs boson [3], the converse, the discovery of the SM Higgs boson, requires the measurements of the

\footnotetext{
*cheung@phys.nthu.edu.tw

†adil.hep@gmail.com

†timluyu@gmail.com

§hsong@konkuk.ac.kr

ywyoon@kias.re.kr
}

Published by the American Physical Society under the terms of the Creative Commons Attribution 4.0 International license. Further distribution of this work must maintain attribution to the author(s) and the published article's title, journal citation, and DOI. Funded by SCOAP ${ }^{3}$. other remaining couplings. At the high-luminosity LHC (HL-LHC), the Higgs trilinear self-coupling $\lambda_{h h h}$ and the Higgs coupling to a muon pair are to be observed $[4,5]$. As the Higgs self-interaction is the key to understand electroweak symmetry breaking, vacuum stability, and electroweak phase transition, many theorists are interested more in $\lambda_{h h h}$. At the LHC, the Higgs boson pair production via gluon fusion, simply called the di-Higgs process, is known to offer a direct probe to $\lambda_{\text {hhh }}$ [5-9].

In the SM, the di-Higgs process receives the contributions from the triangle and box diagrams through the top and bottom quarks $[10,11]$. The triangle diagram is solely mediated by the Higgs boson in $s$-channel, providing the connection to $\lambda_{h h h}$. Under the assumption that the Higgs boson couplings to gauge bosons and fermions are SMlike, there are three main ways to accommodate new physics (NP) effects on $g g \rightarrow h h$. The first is the resonant production of the Higgs boson pair through a new scalar boson or the spin-2 Kaluza-Klein graviton in the RandallSundrum model [12-18]. The second is an anomalous Higgs trilinear self-coupling [19-22], parametrized by the 
Higgs coupling modifier $\kappa_{\lambda} \equiv \lambda_{h h h} / \lambda_{h h h}^{\mathrm{SM}}$. The third is to introduce new colored particles in the triangle and box diagrams [23-28]. ${ }^{1}$

Since resonant Higgs boson pair production can be identified through a peak in the distribution of the invariant mass of the Higgs boson pair, experimentalists present the di-Higgs results in two categories, nonresonant and resonant ones $[35,36]$. And the nonresonant result is usually translated into the limit on $\kappa_{\lambda}$ such that the latest one is $-5.0<\kappa_{\lambda}<12.0$ at $95 \%$ confidence level [35]. However, there is another nonresonant NP effect, the loop-induced effect from new colored particles. In the optimistic case where we shall observe some excesses on nonresonant diHiggs process, the key question is then how to distinguish the loop-induced effects from the anomalous $\lambda_{h h h}$ effects.

We observe that a unique feature of the loop-induced effects is the presence of the threshold ${ }^{2}$ : any new heavy particle $\mathcal{F}$ in the loop would yield a bump structure in the invariant mass distribution of the Higgs pair at $M_{h h} \simeq 2 M_{\mathcal{F}}$. A naive parton level kinematics predicts an associated bump at $p_{T}^{h} \simeq M_{\mathcal{F}}$ when the longitudinal motion is soft. In the literature, the bumps of the threshold origin in the distributions of $M_{h h}$ [24,26,41] and $p_{T}^{h}$ [25] have been separately studied. However, the correlation of two bumps at high scale is a distinguishing point of the loop-induced effects, which the anomalous $\lambda_{h h h}$ effects cannot mimic. And the next issue is whether we can really see the correlated bumps at the level of full HL-LHC simulation.

For the comparative study, we will begin with the assumption of $\sigma_{\mathrm{NP}} / \sigma_{\mathrm{SM}}(g g \rightarrow h h) \simeq 3$ as an illustrative example. An immediate question arises whether loop effects can yield such a large enhancement without significantly affecting the single Higgs production rate. Actually, the loop-induced effects from SUSY particles $[23,24,27,28]$, new fermions along with a composite Higgs boson [41], or fermionic top partners [26,42] yield $\sigma_{\mathrm{NP}} / \sigma_{\mathrm{SM}}(g g \rightarrow h h) \lesssim 2 .^{3}$ In the type-II two-Higgs-doublet model (2HDM) [43] with vectorlike quarks (VLQs), we shall show that $\sigma_{\mathrm{NP}} / \sigma_{\mathrm{SM}}(g g \rightarrow h h) \simeq 3$ is possible in the wrong-sign limit $\left(\kappa_{t}=-\kappa_{b}=1\right)$ [44-48]. Vectorlike quarks, which appear in many new physics models [4964] and fit well with the Higgs precision data [65,66], are introduced for new loop-induced effects. The consistency of the model with theoretical and experimental constraints, especially from electroweak oblique parameters and Higgs precision data, will be explicitly shown through the

\footnotetext{
${ }^{1}$ We note other NP effects on nonresonant di-Higgs process through anomalous model-independent dimension-six effective operators [29-32] or through anomalous top Yukawa couplings $[33,34]$.

${ }^{2}$ This is to be distinguished from the threshold regimes, corresponding to $M_{h h} \simeq 2 m_{h}$, where the triangle diagram is compatible with the box diagram [37-40].

${ }^{3}$ In a NP model with light color-octet scalars [25], $\sigma_{\mathrm{NP}} / \sigma_{\mathrm{SM}}(g g \rightarrow h h) \sim \mathcal{O}\left(10^{3}\right)$ is possible.
}

comprehensive phenomenology study. We will perform a full analytic calculation of the VLQ contributions to the form factors of the di-Higgs process and the HL-LHC simulation in the $b \bar{b} b \bar{b}$ and $b \bar{b} \gamma \gamma$ final states. The correlated bumps around $M_{h h} \simeq 2 M_{\mathrm{VLQ}}$ and $p_{T}^{h} \simeq M_{\mathrm{VLQ}}$ are to be shown as one of the characteristic features of the loopinduced effects on the di-Higgs process. These are our main results.

The paper is organized in the following way. In Sec. II, we begin with summarizing the characteristics of Higgs boson pair production from gluon fusion. Focusing on the nonresonant case, we parametrize the NP effects and motivate our model, the VLQ-2HDM. In Sec. III, we briefly review the VLQ-2HDM and suggest an ansatz for vanishing Peskin-Takeuchi parameter $\hat{T}$ [67]. In Sec. IV, we present the parton-level study of the VLQ-2HDM effects on the di-Higgs process, including the full analytic calculation of the form factors from new VLQs. For a benchmark point in the exact wrong-sign limit, we show the differences among different NP models in the kinematic distributions of $p_{T}^{h}$ and $M_{h h}$. Section V deals with the full HL-LHC simulations of three NP models and the SM in the $b \bar{b} b \bar{b}$ and $b \bar{b} \gamma \gamma$ final states, focusing on the double differential cross sections. Section VI contains our conclusions.

\section{NONRESONANT DI-HIGGS PRODUCTION FROM GLUON FUSION}

Gluon-gluon fusion production of a pair of Higgs bosons is a loop-induced process from two types of Feynman diagrams, triangle and box diagrams: see Fig. 1. In the SM, the top quark makes major contribution to the process in both diagrams. Since the triangle diagram is solely mediated by the Higgs boson in $s$-channel, the Higgs trilinear coupling can be probed. The partonic differential cross section to leading order is [11]

$$
\begin{aligned}
\frac{d \hat{\sigma}^{\mathrm{SM}}(g g \rightarrow h h)}{d \hat{t}}= & \frac{G_{F}^{2} \alpha_{s}^{2}}{256(2 \pi)^{3}}\left[\mid \lambda_{h h h}^{\mathrm{SM}} \frac{v}{\hat{s}-m_{h}^{2}+i m_{h} \Gamma_{h}} F_{\Delta}\right. \\
& \left.+\left.F_{\square}\right|^{2}+\left|G_{\square}\right|^{2}\right],
\end{aligned}
$$

where $\lambda_{h h h}^{\mathrm{SM}}\left(=3 m_{h}^{2} / v\right)$ is the Higgs trilinear self-coupling, and the expressions for $F_{\Delta}, F_{\square}$, and $G_{\square}$ are referred to Ref. [11]. In the low-energy theorem (LET) where $m_{Q}^{2} \gg \hat{s}-m_{h}^{2}$, equivalently in the large VLQ mass limit, the form factors are simplified as

$$
F_{\Delta}^{\mathrm{LET}} \simeq \frac{2}{3}, \quad F_{\square}^{\mathrm{LET}} \simeq-\frac{2}{3}, \quad G_{\square}^{\mathrm{LET}} \simeq 0,
$$

which clearly show the destructive interference between the triangle and box diagrams. Special attention is required when using Eq. (2). Although they are useful in estimating the total production cross section, the kinematic distributions 

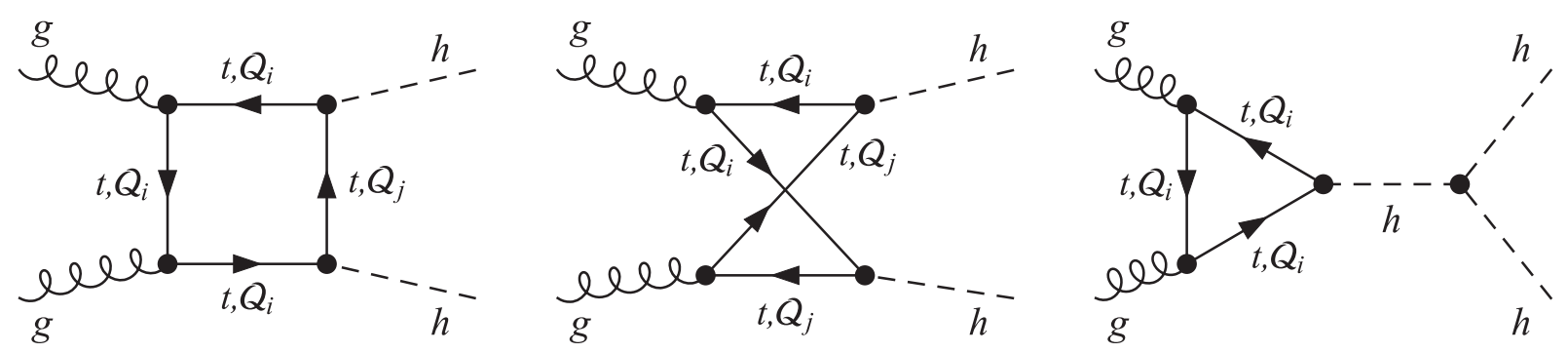

FIG. 1. Representative Feynman diagrams for the di-Higgs process via gluon-gluon fusion at the LHC. In addition to the SM top quark, new VLQs $\left(\mathcal{Q}_{i}=\mathcal{U}_{1,2}, \mathcal{D}_{1,2}\right)$ also contribute to the triangle and box diagrams.

based on the approximated form factors are significantly different from the exact calculations, especially in the high $p_{T}$ region [42].

For illustrative purpose, we assume that the di-Higgs process is observed at the HL-LHC with the total cross section about three times as large as the SM prediction ${ }^{4}$ :

$$
\left.\frac{\sigma(g g \rightarrow h h)_{\mathrm{NP}}}{\sigma(g g \rightarrow h h)_{\mathrm{SM}}}\right|_{14 \mathrm{TeV}} \simeq 3
$$

We further suppose that the possibility of resonant Higgs boson pair production is ruled out from the study of the invariant-mass distribution of two Higgs bosons (via e.g., $h h \rightarrow b \bar{b} \gamma \gamma$ [68]). For nonresonant sources of Eq. (3), we consider the following two kinds of NP effects:

(i) $\kappa_{\lambda}=-0.5,5.5$;

(ii) new VLQs.

Two NP effects are effectively parametrized by $\kappa_{\lambda}, \delta_{\Delta}, \delta_{\square}$, and $\delta_{\square}^{\prime}$, which change the partonic differential cross section into

$$
\begin{aligned}
& \frac{d \hat{\sigma}(g g \rightarrow h h)_{\mathrm{NPe}}}{d \hat{t}} \\
& =\frac{G_{F}^{2} \alpha_{s}^{2}}{256(2 \pi)^{3}}\left[\left|\kappa_{\lambda} \frac{3 m_{h}^{2}}{\hat{s}-m_{h}^{2}}\left(F_{\Delta}+\frac{2}{3} \delta_{\Delta}\right)+\left(F_{\square}-\frac{2}{3} \delta_{\square}\right)\right|^{2}\right. \\
& \left.\quad+\left|G_{\square}+\delta_{\square}^{\prime}\right|^{2}\right] .
\end{aligned}
$$

For the case (i), we take the SM except for the Higgs trilinear self-coupling. Let us make some comments on the values of $\kappa_{\lambda}=-0.5$ and $\kappa_{\lambda}=5.5$, which are chosen, as simple representative numbers, to approximately satisfy $\sigma_{\mathrm{NP}} / \sigma_{\mathrm{SM}}(g g \rightarrow h h) \simeq 3$. Our calculation of the signal in what follows is at leading order. However, the $K$-factor in the SM is not only quite large like 1.9 at NNLO but also significantly varying with the transverse momentum of the Higgs boson [69]. Without a reliable NLO calculation in the NP model, tuning the value of $\kappa_{\lambda}$ to exactly get $\sigma_{\mathrm{NP}} /\left.\sigma_{\mathrm{SM}}\right|_{\mathrm{LO}}=3$ is not of much importance. Moreover, our main results rely on the shapes of kinematic

\footnotetext{
${ }^{4}$ The factor of three is just a reference value for illustration.
}

distributions, rather than the total cross section. For the case (ii), we extend the SM quark sector by introducing new heavy VLQs. ${ }^{5}$ Of course, there is a possibility that both (i) and (ii) occur simultaneously. Since the combined effect is very different according to the relative contributions from the case (i) and (ii), it is troublesome to quantify the result. We do not consider the mixed case in this work.

One of the most important factors when considering the case (ii) is the correlation between the di-Higgs and single-Higgs processes. If new VLQs contribute to the di-Higgs triangle diagram, they cannot avoid contributing to the same single-Higgs triangle diagram. Since the current Higgs precision data strongly prefer the SM-like Higgs boson, we need to break the correlation in order to enhance the di-Higgs production rate. We find that the key is nonSM Higgs couplings to fermions, which demands an extension of the Higgs sector. In this regard, we consider a 2HDM with the VLQs in two limiting cases, the alignment limit [71-75] (for the SM-like Higgs couplings) and the exact wrong-sign limit [44,47] (for non-SM Higgs couplings).

\section{BRIEF REVIEW OF THE 2HDM WITH VLQS}

We consider a 2HDM with VLQs, simply the VLQ2HDM. The SM Higgs sector is extended by introducing two complex scalar fields, $\Phi_{1}$ and $\Phi_{2}$. The fermion sector also has new field components, two additional $S U(2)_{L^{-}}$ doublet VLQs $\left(\mathcal{Q}_{L, R}\right)$ and four $S U(2)_{L}$-singlet VLQs $\left(\mathcal{U}_{L, R}\right.$ and $\left.\mathcal{D}_{L, R}\right)$ :

two Higgs doublets: $\Phi_{i}=\left(\begin{array}{c}w_{i}^{+} \\ \frac{v_{i}+h_{i}+i \eta_{i}}{\sqrt{2}}\end{array}\right), \quad(i=1,2)$,

$$
\text { VLQ doublets: } \mathcal{Q}_{L}=\left(\begin{array}{c}
\mathcal{U}_{L}^{\prime} \\
\mathcal{D}_{L}^{\prime}
\end{array}\right), \quad \mathcal{Q}_{R}=\left(\begin{array}{c}
\mathcal{U}_{R}^{\prime} \\
\mathcal{D}_{R}^{\prime}
\end{array}\right) \text {, }
$$

VLQ singlets: $\mathcal{U}_{L}, \mathcal{U}_{R}, \mathcal{D}_{L}, \mathcal{D}_{R}$,

where the vacuum expectation values $v_{1}$ and $v_{2}$ satisfy $v=\sqrt{v_{1}^{2}+v_{2}^{2}} \simeq 246 \mathrm{GeV}$ and define $\tan \beta \equiv t_{\beta}=v_{2} / v_{1}$.

\footnotetext{
${ }^{5}$ New chiral fermions, even in the $2 \mathrm{HDM}$, are excluded by the Higgs precision data and the resonance searches in the $Z Z$ and $W^{+} W^{-}$channel $[47,70]$.
} 
In what follows, we use the shorthand notation of $s_{x}=\sin x, c_{x}=\cos x$, and $t_{x}=\tan x$ for simplicity.

In order to avoid tree-level flavor changing neutral currents, a discrete $Z_{2}$ symmetry is imposed under which $\Phi_{1} \rightarrow \Phi_{1}$ and $\Phi_{2} \rightarrow-\Phi_{2}$ [76,77]. According to the $Z_{2}$ parities of the fermions, there are four types in the 2HDM: type-I, type-II, type-X, and type-Y [78]. In this work, we focus on type-II since only it allows the wrong-sign limit, which will offer our key benchmark point. The most general scalar potential with $C P$ invariance is written as

$$
\begin{aligned}
V_{\Phi}= & m_{11}^{2} \Phi_{1}^{\dagger} \Phi_{1}+m_{22}^{2} \Phi_{2}^{\dagger} \Phi_{2}-m_{12}^{2}\left(\Phi_{1}^{\dagger} \Phi_{2}+\text { H.c. }\right) \\
& +\frac{1}{2} \lambda_{1}\left(\Phi_{1}^{\dagger} \Phi_{1}\right)^{2}+\frac{1}{2} \lambda_{2}\left(\Phi_{2}^{\dagger} \Phi_{2}\right)^{2}+\lambda_{3}\left(\Phi_{1}^{\dagger} \Phi_{1}\right)\left(\Phi_{2}^{\dagger} \Phi_{2}\right) \\
& +\lambda_{4}\left(\Phi_{1}^{\dagger} \Phi_{2}\right)\left(\Phi_{2}^{\dagger} \Phi_{1}\right)+\frac{1}{2} \lambda_{5}\left[\left(\Phi_{1}^{\dagger} \Phi_{2}\right)^{2}+\text { H.c. }\right],
\end{aligned}
$$

where $m_{11}^{2}, m_{22}^{2}$, and $\lambda_{1, \ldots, 4}$ are real numbers while $m_{12}^{2}$ and $\lambda_{5}$ can be complex numbers. The $m_{12}^{2}$ term softly breaks the $Z_{2}$ parity. There are five physical Higgs bosons: two $C P$ even scalars (a light Higgs $h$ and a heavy Higgs $H$ ), one $C P$-odd scalar $A$, and two charged Higgs bosons $H^{ \pm}$[58]. These mass eigenstates are related with the weak eigenstates in Eq. (5) as

$$
\begin{aligned}
& \left(\begin{array}{l}
h_{1} \\
h_{2}
\end{array}\right)=\mathbb{R}(\alpha)\left(\begin{array}{c}
H \\
h
\end{array}\right), \quad\left(\begin{array}{c}
w_{1}^{ \pm} \\
w_{2}^{ \pm}
\end{array}\right)=\mathbb{R}(\beta)\left(\begin{array}{c}
G^{ \pm} \\
H^{ \pm}
\end{array}\right), \\
& \left(\begin{array}{l}
\eta_{1} \\
\eta_{2}
\end{array}\right)=\mathbb{R}(\beta)\left(\begin{array}{c}
G^{0} \\
A
\end{array}\right),
\end{aligned}
$$

where $G^{ \pm}$and $G^{0}$ are the Goldstone bosons eaten by $W^{ \pm}$ and $Z$ respectively. The rotation matrix $\mathbb{R}(\theta)$ is

$$
\mathbb{R}(\theta)=\left(\begin{array}{rr}
c_{\theta} & -s_{\theta} \\
s_{\theta} & c_{\theta}
\end{array}\right)
$$

The SM Higgs boson is a linear combination of $h$ and $H$, given by

$$
h_{\mathrm{SM}}=s_{\beta-\alpha} h+c_{\beta-\alpha} H .
$$

Conforming to the SM-like Higgs boson, we consider two limiting cases, the alignment limit and the exact wrongsign (EWS) limit, defined by

$$
\begin{array}{r}
\text { alignment: } \beta-\alpha=\frac{\pi}{2} ; \\
\text { EWS : } \beta+\alpha=\frac{\pi}{2} .
\end{array}
$$

In these limiting cases, the coupling modifiers of $h$ and $H$ are summarized in Table I. Here $\kappa_{i}=g_{i i h} / g_{i i h}^{\mathrm{SM}}$ and $\xi_{i}=$ $g_{i i H} / g_{i i h}^{\mathrm{SM}}$ where $g_{i i h(H)}$ is a typical $h(H)$ coupling constant to gauge bosons and fermions. For the Higgs self-coupling
TABLE I. In the type-II 2HDM, the coupling modifiers of the $C P$-even neutral Higgs bosons, $h$ and $H$, in the alignment limit and the exact wrong-sign (EWS) limit. Here $\kappa_{i}=g_{i i h} / g_{i i h_{\mathrm{SM}}}$ and $\xi_{i}=g_{i i H} / g_{i i h_{\mathrm{SM}}}$ for the typical Higgs coupling $g_{i i h(H)}$. The Higgs trilinear self-coupling modifiers are named by $\kappa_{\lambda}=\lambda_{h h h} / \lambda_{h h h}^{\mathrm{SM}}$ and $\xi_{\lambda}=\lambda_{H h h} / \lambda_{h h h}^{\mathrm{SM}}$. Note that $M^{2} \equiv m_{12}^{2} /\left(s_{\beta} c_{\beta}\right)$.

\begin{tabular}{lcccccc}
\hline \hline Limit & $\kappa_{V}$ & $\xi_{V}$ & $\kappa_{u}$ & $\kappa_{d}$ & $\kappa_{\lambda}$ & $\xi_{\lambda}$ \\
\hline Alignment & 1 & 0 & 1 & 1 & 1 & 0 \\
EWS & $\frac{t_{\beta}^{2}-1}{t_{\beta}^{2}+1}$ & $\frac{2 t_{\beta}}{t_{\beta}^{2}+1}$ & 1 & -1 & $\frac{t_{\beta}^{2}-1}{t_{\beta}^{2}+1}$ & $\frac{t_{\beta}}{1+t_{\beta}^{2}}\left[\frac{4}{3}-\frac{2\left(M_{H}^{2}-2 M^{2}\right)}{3 m_{h}^{2}}\right]$ \\
\hline \hline
\end{tabular}

modifiers, we use the convention $\kappa_{\lambda}=\lambda_{h h h} / \lambda_{h h h}^{\mathrm{SM}}$ and $\xi_{\lambda}=\lambda_{H h h} / \lambda_{h h h}^{\mathrm{SM}}$.

In the alignment limit, $h$ behaves exactly the same as $h_{\mathrm{SM}}$ $\left(\kappa_{i, \lambda}=1\right)$ while the heavy Higgs $H$ is decoupled from the $\operatorname{SM}\left(\xi_{V, \lambda}=0\right)$. Note that the resonant di-Higgs production through $g g \rightarrow H \rightarrow h h$ is absent. In the EWS limit, the coupling of the down-type fermion to the Higgs boson has opposite sign to that of the up-type fermion. Furthermore $\kappa_{V}$ and $\kappa_{\lambda}$ deviate from the SM values and the heavy Higgs boson $H$ is not decoupled. If $t_{\beta} \gg 1$, however, the Higgs couplings become close to the SM ones like $\left|\kappa_{f, V, \lambda}\right| \simeq 1$ and $\xi_{\lambda}$ is also suppressed for large $t_{\beta}$ and can be further suppressed by adjusting the free parameter $m_{12}^{2}$.

The Yukawa Lagrangian for the VLQs is

$$
\begin{aligned}
-\mathcal{L}_{\mathrm{VLQ}}= & M_{\mathcal{Q}} \overline{\mathcal{Q}} \mathcal{Q}+M_{\mathcal{U}} \overline{\mathcal{U}}+M_{\mathcal{D}} \overline{\mathcal{D}} \mathcal{D} \\
& +\left[Y_{\mathcal{D}} \overline{\mathcal{Q}} \Phi_{1} \mathcal{D}+Y_{\mathcal{U}} \overline{\mathcal{Q}}_{2} \mathcal{U}+\text { H.c. }\right]
\end{aligned}
$$

where $\tilde{\Phi}_{i}=i \tau_{2} \Phi_{i}^{*}$ and we assume $Y_{\mathcal{U}(\mathcal{D})}^{L}=Y_{\mathcal{U}(\mathcal{D})}^{R} \equiv Y_{\mathcal{U}(\mathcal{D})}$ for simplicity. The VLQ mass matrices $\mathbb{M}_{\mathcal{D}}$ and $\mathbb{M}_{\mathcal{U}}$ in the basis of $\left(\mathcal{D}^{\prime}, \mathcal{D}\right)$ and $\left(\mathcal{U}^{\prime}, \mathcal{U}\right)$ are

$$
\begin{aligned}
& \mathbb{M}_{\mathcal{D}}=\left(\begin{array}{cc}
M_{\mathcal{Q}} & \frac{1}{\sqrt{2}} Y_{\mathcal{D}} v c_{\beta} \\
\frac{1}{\sqrt{2}} Y_{\mathcal{D}} v c_{\beta} & M_{\mathcal{D}}
\end{array}\right), \\
& \mathbb{M}_{\mathcal{U}}=\left(\begin{array}{cc}
M_{\mathcal{Q}} & \frac{1}{\sqrt{2}} Y_{\mathcal{U}} v s_{\beta} \\
\frac{1}{\sqrt{2}} Y_{\mathcal{U}} v s_{\beta} & M_{\mathcal{U}}
\end{array}\right) .
\end{aligned}
$$

The mass eigenstates are $\left(\mathcal{F}_{1}, \mathcal{F}_{2}\right)^{T}=\mathbb{R}\left(\theta_{\mathcal{F}}\right)\left(\mathcal{F}^{\prime}, \mathcal{F}\right)^{T}$ for $\mathcal{F}=\mathcal{U}, \mathcal{D}$. The VLQ mixing angles are given by

$s_{2 \theta_{\mathcal{D}}}=\frac{\sqrt{2} Y_{\mathcal{D}} v}{M_{\mathcal{D}_{2}}-M_{\mathcal{D}_{1}}} c_{\beta}, \quad s_{2 \theta_{\mathcal{U}}}=\frac{\sqrt{2} Y_{\mathcal{U}} v}{M_{\mathcal{U}_{2}}-M_{\mathcal{U}_{1}}} s_{\beta}$,

where $M_{\mathcal{U}_{1,2}}$ and $M_{\mathcal{D}_{1,2}}$ are mass eigenvalues for the up-type and down-type VLQs, respectively. We parametrize the Higgs couplings to the VLQ mass eigenstates by

$$
-\mathscr{L}_{\mathrm{VLQ}} \supset \sum_{i, j=1,2} h\left[y_{\mathcal{D}_{i}}^{h} \mathcal{D}_{j} \overline{\mathcal{D}}_{i} \mathcal{D}_{j}+y_{\mathcal{U}_{i} \mathcal{U}_{j}}^{h} \overline{\mathcal{U}}_{i} \mathcal{U}_{j}\right]
$$


where for $\mathcal{F}=\mathcal{U}, \mathcal{D}$ they are

$$
\begin{aligned}
& y_{\mathcal{F}_{1} \mathcal{F}_{1}}^{h}=-y_{\mathcal{F}_{2} \mathcal{F}_{2}}^{h}=-\frac{1}{\sqrt{2}} Y_{\mathcal{F}} \xi_{\mathcal{F}}^{h} s_{2 \theta_{\mathcal{F}}}, \\
& y_{\mathcal{F}_{1} \mathcal{F}_{2}}^{h}=y_{\mathcal{F}_{2} \mathcal{F}_{1}}^{h}=\frac{1}{\sqrt{2}} Y_{\mathcal{F}} \xi_{\mathcal{F}}^{h} c_{2 \theta_{\mathcal{F}}} .
\end{aligned}
$$

In type-II, $\xi_{\mathcal{U}}^{h}=c_{\alpha}$ and $\xi_{\mathcal{D}}^{h}=-s_{\alpha}$.

Three major constraints on the VLQ-2HDM are to be discussed. The first one is from the Higgs precision measurements, especially the loop-induced VLQ contributions to $\kappa_{g}: \kappa_{\gamma}$ is less constrained because the $h-\gamma-\gamma$ vertex is mainly from $W^{ \pm}$boson loops. In the presence of VLQs, $\kappa_{g}$ becomes

$$
\kappa_{g}=1+\frac{v}{A_{1 / 2}^{H}\left(\tau_{t}\right)} \sum_{i=1,2} \sum_{\mathcal{F}=\mathcal{U}, \mathcal{D}} \frac{y_{\mathcal{F}_{i}}^{h} \mathcal{F}_{i}}{M_{\mathcal{F}_{i}}} A_{1 / 2}^{H}\left(\tau_{\mathcal{F}_{i}}\right),
$$

where $\tau_{f}=m_{h}^{2} /\left(4 m_{f}^{2}\right)$ and the loop function $A_{1 / 2}^{H}(\tau)$ is referred to Ref. [79]. The relation of $y_{\mathcal{F}_{1} \mathcal{F}_{1}}^{h}=-y_{\mathcal{F}_{2} \mathcal{F}_{2}}^{h}$ in Eq. (15) yields considerable cancelation between the contributions of $\mathcal{F}_{1}$ and $\mathcal{F}_{2}$ to $\kappa_{g}$. The ATLAS combined result of $\kappa_{g}=1.03_{-0.06}^{+0.07}$ [3] is satisfied in most of the parameter space.

The second constraint is from the electroweak precision data, the Peskin-Takeuchi oblique parameters $S, T$, and $U$ $[67,80]$. Based on more general parametrization in terms of $\hat{S}, \hat{T}, W$, and $Y$ [80], we found in the previous work [58] that the most sensitive oblique parameter $\hat{T}$ vanishes in the following ansatz:

zero- $\hat{T}$ ansatz: $M_{\mathcal{U}_{1}}=M_{\mathcal{D}_{1}} \equiv M_{1}, \quad M_{\mathcal{U}_{2}}=M_{\mathcal{D}_{2}} \equiv M_{2}$,

$$
\theta_{\mathcal{U}}=\theta_{\mathcal{D}} \equiv \theta \text {. }
$$

In this ansatz, the up-type and down-type VLQ Yukawa couplings are related as

$$
Y_{\mathcal{U}} s_{\beta}=Y_{\mathcal{D}} c_{\beta}=\frac{s_{2 \theta} \Delta M}{\sqrt{2} v},
$$

where $\Delta M=M_{2}-M_{1}$. Then the Higgs Yukawa couplings to the VLQs in Eq. (15) take the simple forms of

alignment: $y_{\mathcal{U}_{1} \mathcal{U}_{1}}^{h}=y_{\mathcal{D}_{1} \mathcal{D}_{1}}^{h}=-y_{\mathcal{U}_{2} \mathcal{U}_{2}}^{h}=-y_{\mathcal{D}_{2} \mathcal{D}_{2}}^{h}=-\frac{\Delta M}{2 v} s_{2 \theta}^{2}$;

$$
\begin{gathered}
y_{\mathcal{U}_{1} \mathcal{U}_{2}}^{h}=y_{\mathcal{D}_{1} \mathcal{D}_{2}}^{h}=y_{\mathcal{U}_{2} \mathcal{U}_{1}}^{h}=y_{\mathcal{D}_{2} \mathcal{D}_{1}}^{h}=\frac{\Delta M}{2 v} c_{2 \theta} s_{2 \theta} ; \\
\text { EWS: } y_{\mathcal{U}_{1} \mathcal{U}_{1}}^{h}=-y_{\mathcal{D}_{1} \mathcal{D}_{1}}^{h}=-y_{\mathcal{U}_{2} \mathcal{U}_{2}}^{h}=y_{\mathcal{D}_{2} \mathcal{D}_{2}}^{h}=-\frac{\Delta M}{2 v} s_{2 \theta}^{2} ; \\
y_{\mathcal{U}_{1} \mathcal{U}_{2}}^{h}=-y_{\mathcal{D}_{1} \mathcal{D}_{2}}^{h}=y_{\mathcal{U}_{2} \mathcal{U}_{1}}^{h}=-y_{\mathcal{D}_{2} \mathcal{D}_{1}}^{h}=\frac{\Delta M}{2 v} c_{2 \theta} s_{2 \theta} .
\end{gathered}
$$

In the EWS limit, the down-type VLQ Higgs coupling has the opposite sign to the up-type one, while in the alignment limit they are the same. This feature will determine the correlation between the VLQ contributions to the singleHiggs and di-Higgs production rates.

The third constraint is the VLQ mass bound from the direct searches at the LHC [81-102]. The bounds sensitively depend on the decay channels of the lighter VLQs, $\mathcal{U}_{1}$ and $\mathcal{D}_{1}$, which should be into an SM quark. ${ }^{6}$ If they decay only into the third generation quarks, the bounds are strong such that $M_{\mathrm{VLQ}}>1.03 \mathrm{TeV}$ [92]. In order to avoid the strong bound on the VLQ mass, we consider the case where the $\mathcal{U}_{1}$ and $\mathcal{D}_{1}$ decay into the first generation quarks. ${ }^{7}$ The relevant Yukawa interaction Lagrangian in the type-II $2 \mathrm{HDM}$ is

$$
\begin{aligned}
-\mathscr{L}_{\mathrm{Yuk}}^{\mathcal{Q} q}= & y_{d 1} \overline{\mathcal{Q}}_{L} \Phi_{1} d_{R}+y_{d 2} \bar{Q}_{L} \Phi_{1} \mathcal{D}_{R}+y_{u 1} \overline{\mathcal{Q}}_{L} \tilde{\Phi}_{2} u_{R} \\
& +y_{u 2} \bar{Q}_{L} \tilde{\Phi}_{2} \mathcal{U}_{R}+\text { H.c. },
\end{aligned}
$$

where $Q_{L}$ and $u_{R}\left(d_{R}\right)$ are the SM first-generation quark doublet and singlet fields, respectively. Now the decay mode of a lighter VLQ into a light SM quark associated with the Higgs boson is open. Since the couplings to the longitudinally polarized gauge bosons (Goldstone bosons) are enhanced due to the equivalence theorem $[103,104]$, we have

$$
\Gamma\left(\mathcal{U}_{1} \rightarrow h u\right) \simeq \Gamma\left(\mathcal{U}_{1} \rightarrow Z u\right) \simeq \frac{1}{2} \Gamma\left(\mathcal{U}_{1} \rightarrow W^{+} d\right) .
$$

The VLQ search in the light quark channel was carried out in the $W^{ \pm} q$ mode by the ATLAS collaboration [105]. Since the bound of $M_{\mathcal{Q}} \gtrsim 690 \mathrm{GeV}$ is under the assumption of $\mathcal{B}\left(\mathcal{U}_{1} \rightarrow W^{+} d\right)=1$, we reinterpret it by considering Eq. (21) and find $M_{\mathcal{Q}} \gtrsim 600 \mathrm{GeV}$.

The final comment is that the low energy physics requires very small $y_{u i / d i}$. As the Yukawa couplings between the VLQs and the SM quarks, $y_{u i / d i}$ yield offdiagonal elements in the extended quark mass matrix. The minimal flavor violation is no longer valid, resulting in new contributions to the FCNC processes as well as the CKM unitarity violation. In order to evade them, we assume that $y_{u i / d i}$ are very suppressed, like $\sim 10^{-7}$. Note that extremely small $y_{u i / d i}$ do not affect the decays of the lighter VLQs in Eq. (21) because there are no other decay channels. Another concern is whether an extremely small $y_{u_{i} / d_{i}}$ will make the lightest VLQs $\left(\mathcal{U}_{1}\right.$ and $\left.\mathcal{D}_{1}\right)$ long-lived particles and be constrained by the relevant searches at the LHC [106]. However, for $y_{u i / d i} \sim 10^{-7}$, the lighter VLQ decaying as in Eq. (21) is promptly decaying particle under the LHC Run-II trigger and analysis, since it has $c \tau \sim$

\footnotetext{
${ }^{6}$ The heavier VLQs mainly decay into the lighter VLQs associated with the Higgs boson via the Yukawa couplings in Eq. (19).

The decays into the second generation quarks yield practically the same signatures as in the case of the first generation quarks.
} 
$\mathcal{O}(1) \mathrm{mm}$ and thus the constraints from the searches for a long-lived particle through displaced vertices do not apply here.

\section{CHARACTERISTICS OF THE NONRESONANT NP EFFECTS ON THE DI-HIGGS PROCESS}

In this section, we study the phenomenological characteristics of different NP effects on the nonresonant di-Higgs process. First we need to find a reasonable benchmark point in the VLQ-2HDM, satisfying $\sigma_{\mathrm{NP}} / \sigma_{\mathrm{SM}}(g g \rightarrow h h) \simeq 3$ and $\sigma_{\mathrm{NP}} / \sigma_{\mathrm{SM}}(g g \rightarrow h) \simeq 1$ simultaneously. Equation (4), $\sigma_{\mathrm{NP}} / \sigma_{\mathrm{SM}}(g g \rightarrow h h)$ in terms of $\delta_{\Delta}, \delta_{\square}$, and $\delta_{\square}^{\prime}$, will help the exploration. In the alignment limit which guarantees $\kappa_{\lambda}=1$, the ratio at the $14 \mathrm{TeV} \mathrm{LHC}$ is

$$
\begin{aligned}
\left.\frac{\sigma(g g \rightarrow h h)_{\mathrm{NP}}}{\sigma(g g \rightarrow h h)_{\mathrm{SM}}}\right|_{\kappa_{\lambda}=1}= & 1-0.37 \delta_{\Delta}+0.92 \delta_{\square}-0.28 \delta_{\square}^{\prime} \\
& +0.13 \delta_{\Delta}^{2}+1.57 \delta_{\square}^{2}+3.54 \delta_{\square}^{\prime 2} \\
& -0.62 \delta_{\Delta} \delta_{\square},
\end{aligned}
$$

where the NNPDF30_LO parton distribution function set is used [107]. In the EWS limit, $\kappa_{\lambda}$ is slightly deviated from one: for $t_{\beta}=5, \kappa_{\lambda} \simeq 0.92$ and the ratio is

$$
\begin{aligned}
\left.\frac{\sigma(g g \rightarrow h h)_{\mathrm{NP}}}{\sigma(g g \rightarrow h h)_{\mathrm{SM}}}\right|_{\kappa_{\lambda}=0.92}= & 1.06-0.36 \delta_{\Delta}+0.98 \delta_{\square}-0.28 \delta_{\square}^{\prime} \\
& +0.11 \delta_{\Delta}^{2}+1.57 \delta_{\square}^{2}+3.54 \delta_{\square}^{2} \\
& -0.57 \delta_{\Delta} \delta_{\square}
\end{aligned}
$$

We analytically calculate the new form factors with finite VLQ masses, which are almost consistent with the formulas in Ref. [19]. ${ }^{8}$ In order to double-check, we derived the asymptotic behaviors of the new form factors in the LET, and found them completely consistent with those in Ref. [42]. For $M_{\mathcal{F}} \gg 2 m_{h}$, the new form factors are

$$
\begin{aligned}
\delta_{\Delta} \simeq & \sum_{i=1,2}\left[\frac{v}{M_{\mathcal{U}_{i}}} y_{\mathcal{U}_{i} \mathcal{U}_{i}}^{h}+\frac{v}{M_{\mathcal{D}_{i}}} y_{\mathcal{D}_{i} \mathcal{D}_{i}}^{h}\right], \\
\delta_{\square} \simeq & \sum_{i=1,2}\left[\frac{v^{2}}{M_{\mathcal{U}_{i}}^{2}}\left(y_{\mathcal{U}_{i} \mathcal{U}_{i}}^{h}\right)^{2}+\frac{v^{2}}{M_{\mathcal{D}_{i}}^{2}}\left(y_{\mathcal{D}_{i} \mathcal{D}_{i}}^{h}\right)^{2}\right] \\
& +\sum_{\mathcal{F}=\mathcal{U}, \mathcal{D}} \frac{2 v^{2}}{M_{\mathcal{F}_{1}} M_{\mathcal{F}_{2}}}\left(y_{\mathcal{F}_{1} \mathcal{F}_{2}}^{h}\right)^{2}, \\
\delta_{\square}^{\prime} & 0 .
\end{aligned}
$$

\footnotetext{
${ }^{8}$ We found several typos in Ref. [19]. In Eq. (B12), there are three typos: (i) the overall sign in the right-hand side should be $(+)$; (ii) " $-4\left(D_{27[t, t, t, T]}^{(1,2,3)}+\cdots\right.$ ” should be “ $-8\left(D_{27[t, t, t, T]}^{(1,2,3)}+\cdots "\right.$; (iii) “. . - $\left.\left.C_{[t, t, T]}^{(3,4)}\right)\right\}$ ” should be “. $\left.\left.\cdots-\frac{1}{2} C_{[t, t, T]}^{(3,4)}\right)\right\}$ ”. In Eq. (B13), we should replace " $-16\left(\frac{\epsilon_{t}}{\sqrt{2}}\right)\left(\frac{\epsilon_{T}}{\sqrt{2}}\right) m_{t} m_{T}\left(\cdots "\right.$ by " $-32\left(\frac{\epsilon_{t}}{\sqrt{2}}\right) \times$ $\left(\frac{\epsilon_{T}}{\sqrt{2}}\right) m_{t} m_{T}(\cdots "$
}

Adopting the zero- $\hat{T}$ ansatz in Eq. (17), where $y_{\mathcal{U}_{i} \mathcal{U}_{i}}^{h}=$ $y_{\mathcal{D}_{i} \mathcal{D}_{i}}^{h}$ in the alignment limit while $y_{\mathcal{U}_{i} \mathcal{U}_{i}}^{h}=-y_{\mathcal{D}_{i} \mathcal{D}_{i}}^{h}$ in the EWS limit, the NP form factors are further simplified as

$$
\begin{gathered}
\delta_{\Delta}^{\text {zero }-\hat{T}} \simeq \begin{cases}-\frac{(\Delta M)^{2}}{M_{1} M_{2}} s_{2 \theta}^{2} & (\text { alignment }) ; \\
0 & (\text { EWS }) ;\end{cases} \\
\delta_{\square}^{\text {zero- } \hat{T}} \simeq \frac{(\Delta M)^{2}}{M_{1} M_{2}} s_{2 \theta}^{2}+\frac{1}{2} \frac{(\Delta M)^{4}}{M_{1}^{2} M_{2}^{2}} s_{2 \theta}^{4} \quad(\text { alignment \& EWS }) .
\end{gathered}
$$

As shown in Eqs. (22) and (26), the contributions from the box diagrams in both limits are constructive to the SM contribution. Moreover, $\delta_{\square}$ is proportional to the quadratic or quartic terms of the VLQ mass difference $\Delta M$ : we need sizable $\Delta M$ to enhance the di-Higgs production rate. In the alignment limit, large $\Delta M$ also increases $\delta_{\Delta}$ and thus the contribution to the single-Higgs production rate. In the EWS limit, however, $\delta_{\Delta}$ is negligible because of the relation of $y_{\mathcal{U}_{i} \mathcal{U}_{i}}^{h}=-y_{\mathcal{D}_{i} \mathcal{D}_{i}}^{h}$ : see Eq. (19).

The correlations between the di-Higgs production rate and other constraints are summarized in Fig. 2. Over the parameter space $\left(\delta_{\Delta}, \delta_{\square}\right)$, we present the contours of $\sigma_{\mathrm{NP}} / \sigma_{\mathrm{SM}}$ of the di-Higgs process (blue lines) in the VLQ-2HDM for the alignment limit (left panel) and EWS limit (right panel) with $\delta_{\square}^{\prime}=0$ and $t_{\beta}=5$. As can be seen from the slopes of the contours, $\sigma_{\mathrm{NP}} / \sigma_{\mathrm{SM}}$ depends more sensitively on $\delta_{\square}$ than $\delta_{\Delta}$. This is attributed to the larger coefficients of $\delta_{\square}$ and $\delta_{\square}^{2}$ than those of $\delta_{\Delta}$ and $\delta_{\Delta}^{2}$ in Eqs. (22) and (23). The constraints from the electroweak oblique parameter $\hat{T}$ along with the LHC direct searches for the VLQ and the perturbativity of Yukawa couplings are shown by the scatter plots. The red dots are allowed by the oblique parameter $\hat{T}$ at $2 \sigma$ [108], through scanning the parameters over the following range:

$$
\begin{gathered}
M_{\mathcal{U}_{1,2}}, \quad M_{\mathcal{D}_{1,2}}>600 \mathrm{GeV}, \quad \bar{Y}_{\mathcal{U}}\left(\equiv Y_{\mathcal{U}} s_{\beta}\right), \\
\bar{Y}_{\mathcal{D}}\left(\equiv Y_{\mathcal{D}} c_{\beta}\right)<4 \pi .
\end{gathered}
$$

Additionally, we present the results of the zero- $\hat{T}$ ansatz by red lines. Finally we show the $2 \sigma$ exclusion region (grey areas) by the current Higgs precision data of $\kappa_{g}=$ $1.03_{-0.06}^{+0.07}[3]$.

The alignment and EWS limits exhibit very different behaviors. In the alignment limit, the result of the zero- $\hat{T}$ ansatz (red line) shows a strong correlation of $\delta_{\square} \approx-\delta_{\Delta}$. In addition, all of the red dots are closely gathered around the zero- $\hat{T}$ ansatz line. A large $\delta_{\square}$ inevitably leads to a large $\delta_{\Delta}$, which is severely limited by the single-Higgs production rate such as $\left|\delta_{\Delta}\right| \lesssim 0.1$. In the alignment limit, therefore, the current LHC Higgs precision data permit at most $20 \%$ increase in the di-Higgs production rate. In the EWS limit, the zero- $\hat{T}$ ansatz (red line) guarantees $\delta_{\Delta} \simeq 0$ so that the 

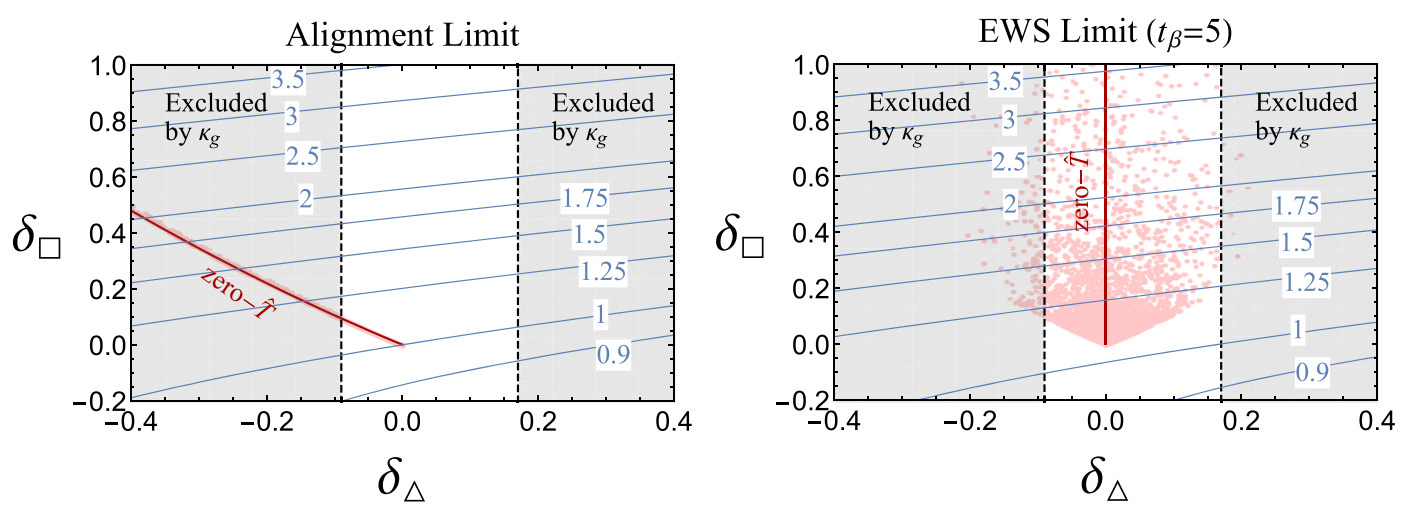

FIG. 2. The VLQ-2HDM prediction of the di-Higgs production rate and various constraints on $\left(\delta_{\Delta}, \delta_{\square}\right)$ in the alignment (left panel) and EWS (right panel) limits. We set $t_{\beta}=5$. The blue contours denote $\sigma_{\mathrm{NP}} / \sigma_{\mathrm{SM}}(g g \rightarrow h h)$ by assuming $\delta_{\square}^{\prime}=0$. The red scatter dots are allowed by the electroweak oblique parameters at $2 \sigma$, the direct LHC search bounds on the VLQ masses, and the perturbativity of the Yukawa coupling. The red lines are the results of the zero- $\hat{T}$ ansatz. The grey regions are excluded by the current measurement on the Higgs coupling modifier $\kappa_{g}$ at $2 \sigma$.

constraint from $\kappa_{g}$ becomes negligible. Relaxing the $\hat{T}$ constraint within $2 \sigma$ (red dots) allows much wider spread of the allowed parameter points in $\left(\delta_{\Delta}, \delta_{\square}\right)$, quite far from the red line.

On account of the overall features in Fig. 2, we take the following benchmark point in the EWS limit for our basic assumption $\sigma_{\mathrm{NP}} / \sigma_{\mathrm{SM}}(g g \rightarrow h h) \simeq 3$ :

benchmark: $\beta+\alpha=\frac{\pi}{2}, \quad t_{\beta}=5$,

$$
M_{1}=600 \mathrm{GeV}, \quad \Delta M=900 \mathrm{GeV}, \quad \theta=0.6 .
$$

Some implications of the benchmark point are to be discussed. First, the contributions from $\mathcal{U}_{2}$ and $\mathcal{D}_{2}$ to the di-Higgs rate are much smaller than those of $\mathcal{U}_{1}$ and $\mathcal{D}_{1}$, below $\sim 1 \%$. Second, we have $\left|y_{\mathcal{Q}_{i} \mathcal{Q}_{i}}^{h}\right| \simeq 1.6$ and $\left|y_{\mathcal{Q}_{1} \mathcal{Q}_{2}}^{h}\right| \simeq$ $0.6\left(\mathcal{Q}_{i}=\mathcal{U}_{i}, \mathcal{D}_{i}\right)$ so that the perturbativity of the VLQ Yukawa couplings is well maintained and the two-loop VLQ corrections on $\delta_{\Delta}$ are expected to be small. Finally, $t_{\beta}=5$ leads to $\kappa_{V}=0.92$, which is still allowed by the recent Higgs data of $\kappa_{Z}>0.88$ and $\kappa_{W}>0.85$ at 95\% C.L. for the case of $\kappa_{V} \leq 1$ [3].

Now we show the $M_{h h}$ (left panel) and $p_{T}^{h}$ (right panel) distributions of the di-Higgs process at the $14 \mathrm{TeV}$ LHC in Fig. 3. We consider the benchmark point of the VLQ2HDM with full calculations of the form factors (black solid line), the VLQ-2HDM with the LET approximation (black dotted line), the SM with $\kappa_{\lambda}=5.5$ (yellow long dashed line) and the SM with $\kappa_{\lambda}=-0.5$ (orange dashed line). As a reference, we also present the SM results (blue solid line). All of the results are at the parton level with the NNLO K-factor $K=1.85$ [69,109-112]. It is clear that the $M_{h h}$ and $p_{T}^{h}$ distributions are very different according to the NP model. For $\kappa_{\lambda}=-0.5$, both $M_{h h}$ and $p_{T}^{h}$ distributions slightly shift toward the lower region, compared with those in the SM. If $\kappa_{\lambda}=5.5$, the shift to the left is much more significant: the peak positions in both distributions move about $100 \mathrm{GeV}$. In the VLQ-2HDM, both differential cross sections show very distinctive features. First, they decrease slowly as $M_{h h}$ or $p_{T}^{h}$ increases. It is because the box diagrams from VLQs, which do not have the $1 / \hat{s}$ suppression at the amplitude level as in Eq. (4), mainly enhance the di-Higgs process. Most importantly, we do see the threshold effects appear as the bump structures starting at the positions $M_{h h} \simeq 2 M_{1}$ and $p_{T}^{h} \simeq M_{1}$. Furthermore, the bumps lift both distributions up in the high- $M_{h h}$ and high- $p_{T}$ regions. Note that if we use the approximated form factors for the VLQ-2HDM (black dotted lines), the bump structures disappear.

In order to quantitatively show the different $p_{T}^{h}$ shift according to the NP model, we calculate the ratio of the di-Higgs production cross section after $p_{T}^{h}>300 \mathrm{GeV}$ cut to the total cross section:

$$
\begin{aligned}
& \frac{\sigma\left(g g \rightarrow h h ; p_{T}^{h}>300 \mathrm{GeV}\right)}{\sigma_{\mathrm{tot}}(g g \rightarrow h h)} \\
& \quad= \begin{cases}6.1 \%, & (\mathrm{SM}) \\
14.5 \%, & \left(\text { VLQ-2HDM with } M_{1}=600 \mathrm{GeV}\right) \\
3.2 \%, & \left(\kappa_{\lambda}=-0.5\right) \\
1.2 \%, & \left(\kappa_{\lambda}=5.5\right)\end{cases}
\end{aligned}
$$

The results clearly show that only the VLQ-2HDM events considerably survive after the high $p_{T}^{h}$ cut. This feature of the VLQ-2HDM is advantageous to suppress the background of the di-Higgs process $[68,113]$. All of the SM backgrounds yield lower $p_{T}^{h}$ distributions than the SM diHiggs process (see Fig. 4 in Ref. [68]).

Before presenting the full HL-LHC simulation in the next section, we discuss some other aspects of the 

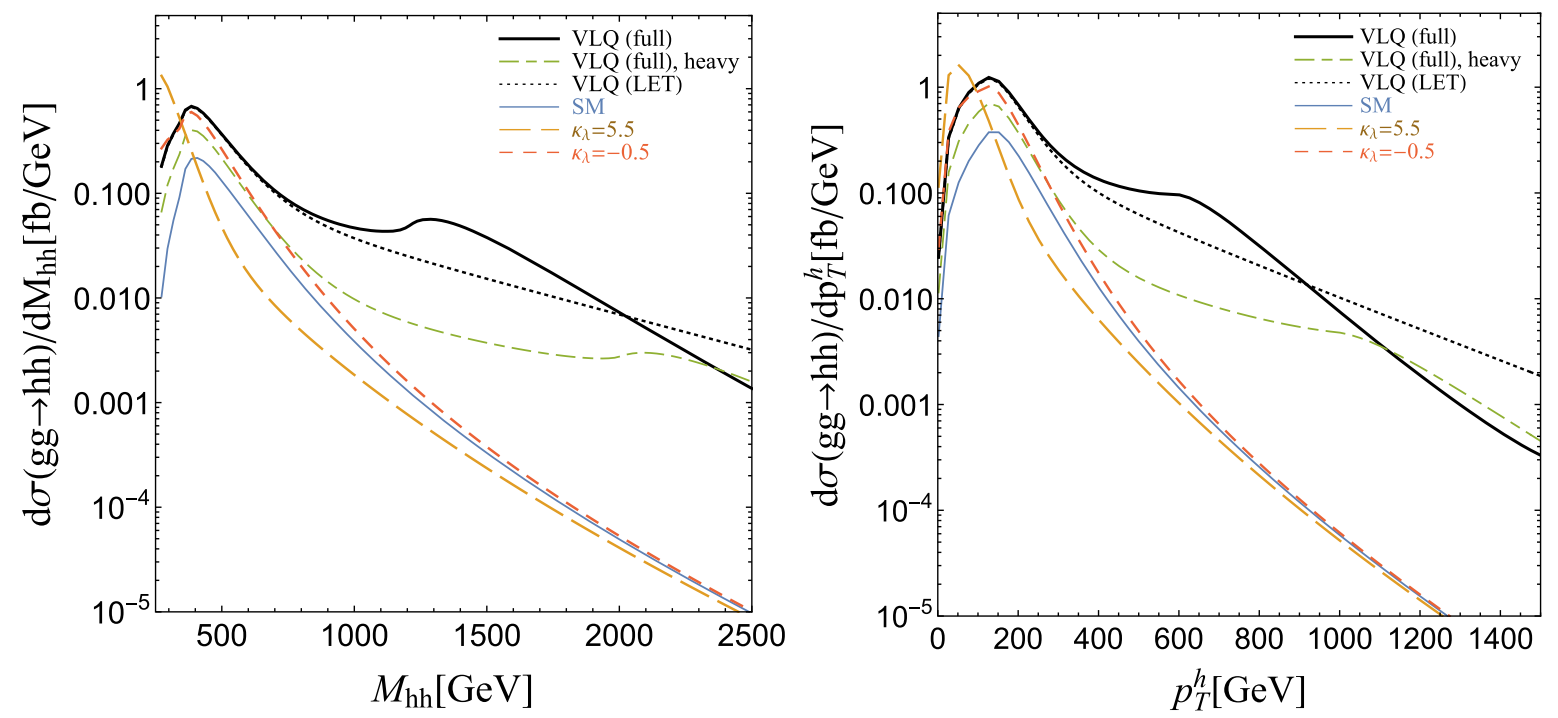

FIG. 3. The distributions of the invariant mass of the Higgs-boson pair (left panel) and those of the transverse momentum of one of the Higgs bosons (right panel) for the parton level $g g \rightarrow h h$ process at the 14 TeV LHC. We consider the VLQ-2HDM in Eq. (28) with full calculations of the form factors (black solid line), the VLQ-2HDM with the low energy theorem approximation (black dotted line), the SM (blue solid line), the SM with $\kappa_{\lambda}=5.5$ (brown long dashed line) and $\kappa_{\lambda}=-0.5$ (orange dashed line). We also show the heavy VLQ case with $M_{1}=1 \mathrm{TeV}$ and $\Delta M=1 \mathrm{TeV}$ (green line).

loop-induced VLQ effects. The first is the concern about whether the bump structures shall remain if the VLQ is heavier than $600 \mathrm{GeV}$. To settle the concern, we consider $M_{1}=1 \mathrm{TeV}$ and $\Delta M=1 \mathrm{TeV}$ while keeping the other parameters as the benchmark in Eq. (28). The total cross section is about 2.3 times as large as the SM cross section, less than the $M_{1}=600 \mathrm{GeV}$ case but not negligible. The $M_{h h}$ and $p_{T}^{h}$ distributions are illustrated in Fig. 3 by green lines, which definitely show two correlated bumps starting at $M_{h h} \simeq 2 M_{1}$ and $p_{T}^{h} \simeq M_{1}$. As the bumps appear at higher scale, the signal rate is inevitably smaller than that for the $M_{1}=600 \mathrm{GeV}$ case, almost by one order of magnitude. However, the long tail in the high $p_{T}^{h}$ region, towed by the bump, still remains. Similarly in Eq. (29), we calculate the cross section after $p_{T}^{h}>500 \mathrm{GeV}$ cut, yielding

$$
\begin{aligned}
& \frac{\sigma\left(g g \rightarrow h h ; p_{T}^{h}>500 \mathrm{GeV}\right)}{\sigma_{\mathrm{tot}}^{\mathrm{SM}}(g g \rightarrow h h)} \\
& = \begin{cases}0.67 \%, & (\mathrm{SM}) \\
8.4 \%, & \left(\mathrm{VLQ}-2 \mathrm{HDM} \text { with } M_{1}=1 \mathrm{TeV}\right) .\end{cases}
\end{aligned}
$$

Since almost all of the backgrounds of the di-Higgs process are known to disappear after the cut of $p_{T}^{h}>400 \mathrm{GeV}$ and $M_{h h}>800 \mathrm{GeV}$ (see Figs. 7 and 17 in Ref. [113]), we expect that the $1 \mathrm{TeV}$ VLQ effects can be indirectly probed through very high $p_{T}^{h}$ cut, provided that there is enough statistics.

The second concern is about whether the shift into high $M_{h h}$ and high $p_{T}^{h}$ from the correlated bumps is a unique feature of the loop-induced effects. The first candidate as a mimic is a wide resonance $X$ : it is known to yield similar bumps when $M_{X} \simeq 2 M_{\mathcal{Q}}$ [114]. As an example, we consider a resonant di-Higgs process, $g g \rightarrow H \rightarrow h h$, in a 2HDM without VLQs. In order to allow nonvanishing vertex of $H-h-h$, we set $s_{\beta-\alpha}=0.95$, which should not be confused with the 2HDM-VLQ case where the contribution of $H$ to the di-Higgs process is negligible. In Fig. 4, we present the distributions of $M_{h h}$ (left panel) and $p_{T}^{h}$ (right panel) of $g g \rightarrow H \rightarrow h h$, for $\Gamma_{H}^{\text {tot }}=0.01 M_{H}$ (blue lines), $\Gamma_{H}^{\text {tot }}=0.1 M_{H}$ (red lines), and $\Gamma_{H}^{\text {tot }}=0.5 M_{H}$ (green lines). The results in the 2HDM-VLQ benchmark case (black lines) are also presented for comparison. Although both the wide resonance and the loop-induced effect similarly produce correlated bumps, the shapes are significantly different. In the $M_{h h}$ distribution, the excess from a wide resonance is localized around $M_{h h}=M_{X}$, generating a (wide) peak standing alone. On the contrary, the bumps from the loop-induced effects look like a low hill, lifting almost the whole high $M_{h h}$ region. The $p_{T}^{h}$ distribution shows this difference starkly: the excess from a wide resonance suddenly falls down from the peak position while that from the VLQs yields a long tail in high $p_{T}^{h}$ region.

The final question is the distinction from the effect of a modified top quark Yukawa coupling $\left(\kappa_{t} \neq 1\right)$ on the diHiggs process [115]. The current ATLAS combined result is $\kappa_{t}=1.09_{-0.10}^{+0.15}$ [3] and the expected precision for $\kappa_{t}$ at the HL-LHC is $7-10 \%$ [116-118]. For $\kappa_{t}=1.1$, we found that the total production cross section of the di-Higgs process is about 1.5 times as large as the SM prediction but the shapes of both $d \sigma / d M_{h h}(g g \rightarrow h h)$ and $d \sigma / d p_{T}^{h}(g g \rightarrow h h)$ are almost the same as in the SM. In particular, the peak positions in both distributions remain intact. In summary, 

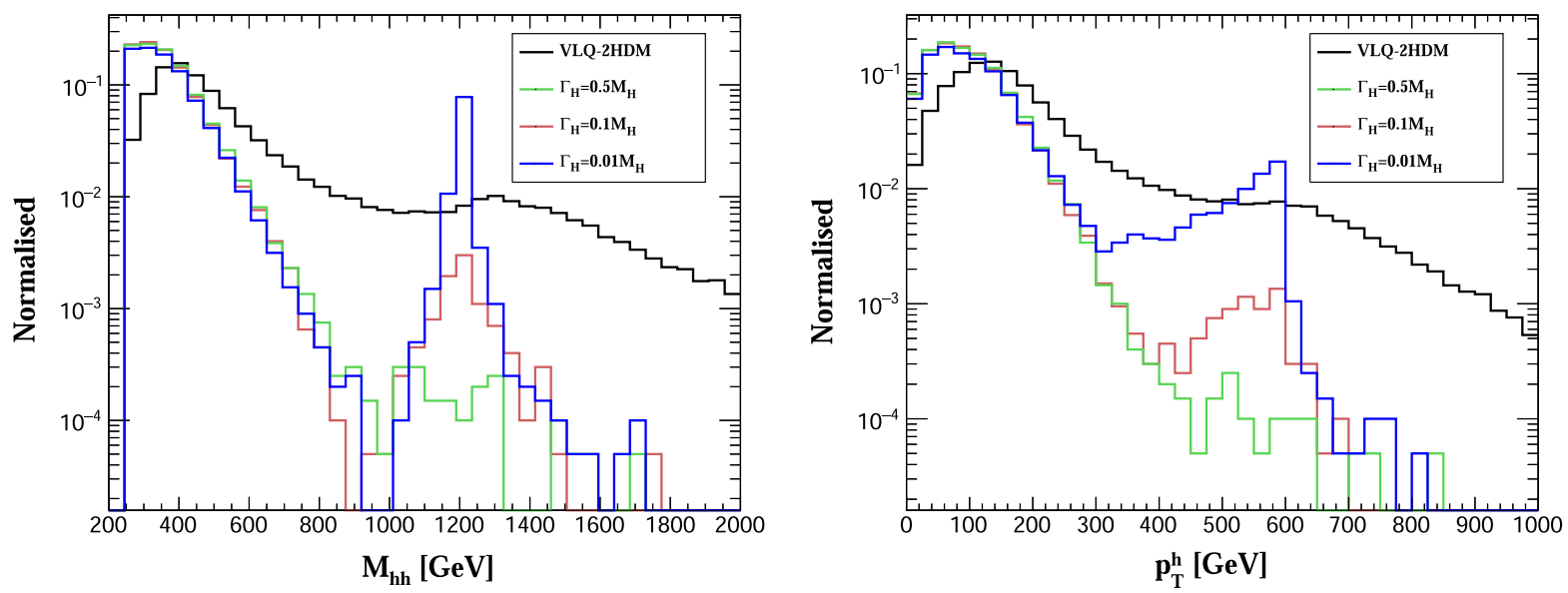

FIG. 4. The distributions of $M_{h h}$ (left panel) and $p_{T}^{h}$ (right panel) at $14 \mathrm{TeV}$ LHC for the resonant di-Higgs process, $g g \rightarrow H \rightarrow h h$, in a $2 \mathrm{HDM}$ without VLQs. As setting $t_{\beta}=1, s_{\beta-\alpha}=0.95$, and $M_{H}=1.2 \mathrm{TeV}$, we consider three cases of $\Gamma_{H}^{\mathrm{tot}}=0.01 M_{H}$ (blue lines), $\Gamma_{H}^{\mathrm{tot}}=0.1 M_{H}$ (red lines), and $\Gamma_{H}^{\mathrm{tot}}=0.5 M_{H}$ (green lines). The black lines correspond to the 2HDM-VLQ benchmark case.

the claimed characteristic, the excess in the high scale regime, is indeed an efficient probe for the loop-induced effects on the di-Higgs process.

\section{SIMULATIONS, EVENT SELECTIONS, AND ANALYSIS AT THE 14 TEV HL-LHC}

As one of the most challenging and significant processes to observe at the LHC, the di-Higgs process has been intensively studied at the state-of-art level. Theoretically the total production cross section was calculated at next-tonext-to-next-to-leading order $\left(\mathrm{N}^{3} \mathrm{LO}\right)$ in the infinite topquark mass limit and the next-to-leading order (NLO) with full top-quark mass dependence [109,110,119-121]. The search strategies to maximize the discovery sensitivity have been suggested for different decay channels such as $b \bar{b} b \bar{b}$ [122], $b \bar{b} \gamma \gamma$ [68,123], and $b \bar{b} W W^{(*)}[124,125]$. On the experimental side, the ATLAS $[35,126]$ and CMS collaborations [36] have performed the search, in different final states such as $b \bar{b} b \bar{b}$ [127-131], $b \bar{b} W W^{(*)}$ [132,133], $b \bar{b} \tau^{+} \tau^{-}$[134], $b \bar{b} \gamma \gamma$ [128,135-137], $\gamma \gamma W W^{(*)}$ [138,139], and $W W^{(*)} W W^{(*)}[140]$.

In this section, we present the full collider simulation of the signals in two final states, $h h \rightarrow b \bar{b} b \bar{b}$ and $h h \rightarrow b \bar{b} \gamma \gamma$. The $4 b$ final state has the advantage of the largest branching ratio of $\mathcal{B}(h h \rightarrow 4 b) \sim 1 / 3$, which has the second-highest sensitivity next to the $b \bar{b} \tau \tau$ final state [35]. Although the major backgrounds from QCD multijet processes $(j j b b$, $j j j j$, and $b b b b)$ and $t \bar{t}$ production are overwhelming, ${ }^{9}$ the dedicated studies on the state-of-the-art multivariate techniques show that the SM di-Higgs process can have a signal significance of $S / \sqrt{B} \simeq 3$ at the HL-LHC

\footnotetext{
${ }^{9}$ The backgrounds from $Z / W+$ jets are extremely suppressed compared to these two especially when the $4 \mathrm{~b}$-tagged jets trigger is applied with $p_{T}^{b}>40 \mathrm{GeV}$.
}

[115,122,141,142]. Another important final state, $b \bar{b} \gamma \gamma$, benefits from clean signal extraction because of a good diphoton invariant mass resolution, despite much smaller branching ratio $\mathcal{B}(h h \rightarrow b \bar{b} \gamma \gamma) \simeq 2.6 \times 10^{-3}$. Although we do not make a full signal-to-background selection analysis here, the correlations among the key observables of the diHiggs process may help in designing new search strategies for the possibility of having VLQs.

The signal events are generated at leading order by using MadGraph5_aMC@NLO $[143,144]$ in the SM, the VLQ$2 \mathrm{HDM}$, the SM with $\kappa_{\lambda}=-0.5$, and $\kappa_{\lambda}=5.5$. The VLQ$2 \mathrm{HDM}$ model file in the UFO format is obtained from modifying an existing 2HDM model file by adding the new contributions of VLQs in the loop, without resort to effective vertices. We thoroughly checked the UFO file by comparing various results with the analytic calculations at parton level. All of the VLQ-2HDM results in this section are based on the benchmark point in Eq. (28). We have chosen the renormalization and factorization scales to be twice the mass of the SM Higgs boson. We employ the NNPDF30_LO PDF set with $\alpha_{s}\left(M_{Z}\right)=0.118$ [107]. The generated events are passed to PYTHIA8 v8.2.43 [145] for parton showering and hadronization, without multipleparton interactions. We use DELPHES 3.4.2 as a fast detector simulation [146] with the ATLAS template. Jets are clustered using the anti- $k_{T}$ algorithm [147] with a jet radius of $R=0.4$ as implemented in FastJet [148].

\section{A. $b \bar{b} b \bar{b}$ final state}

For the $b \bar{b} b \bar{b}$ final state, we follow the ATLAS analysis strategy [127]. We start the event selection by requiring the presence of at least four $b$-jets with $p_{T}^{b}>40 \mathrm{GeV}$ and $\left|\eta^{b}\right|<2.5$. The four leading $b$-jets, ordered by the transverse momentum of each $b$ jet, are used to form two separate dijets: two $b$-jets with the angular distance $\left(\Delta R=\sqrt{\Delta \eta^{2}+\Delta \phi^{2}}\right)$ smaller than 1.5 are identified as 

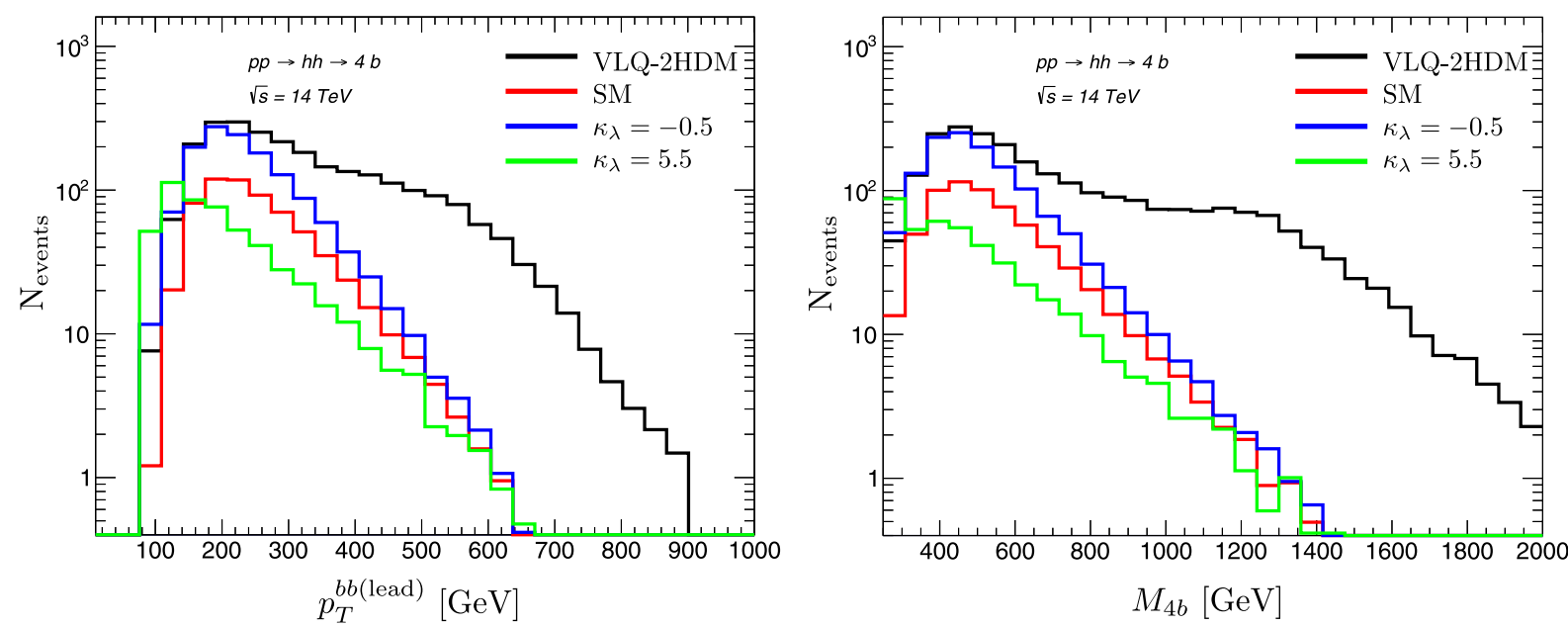

FIG. 5. The expected number of events, after the basic selection, as a function of the transverse momentum of the leading dijet (left panel) and the invariant mass of four leading $b$-jets (right panel) for $g g \rightarrow h h \rightarrow b \bar{b} b \bar{b}$ at the $14 \mathrm{TeV}$ LHC with the total integrated luminosity $3000 \mathrm{fb}^{-1}$. The distributions are for the VLQ-2HDM (black line), the SM (red), the SM with $\kappa_{\lambda}=-0.5$ (blue), and $\kappa_{\lambda}=5.5$ (green).
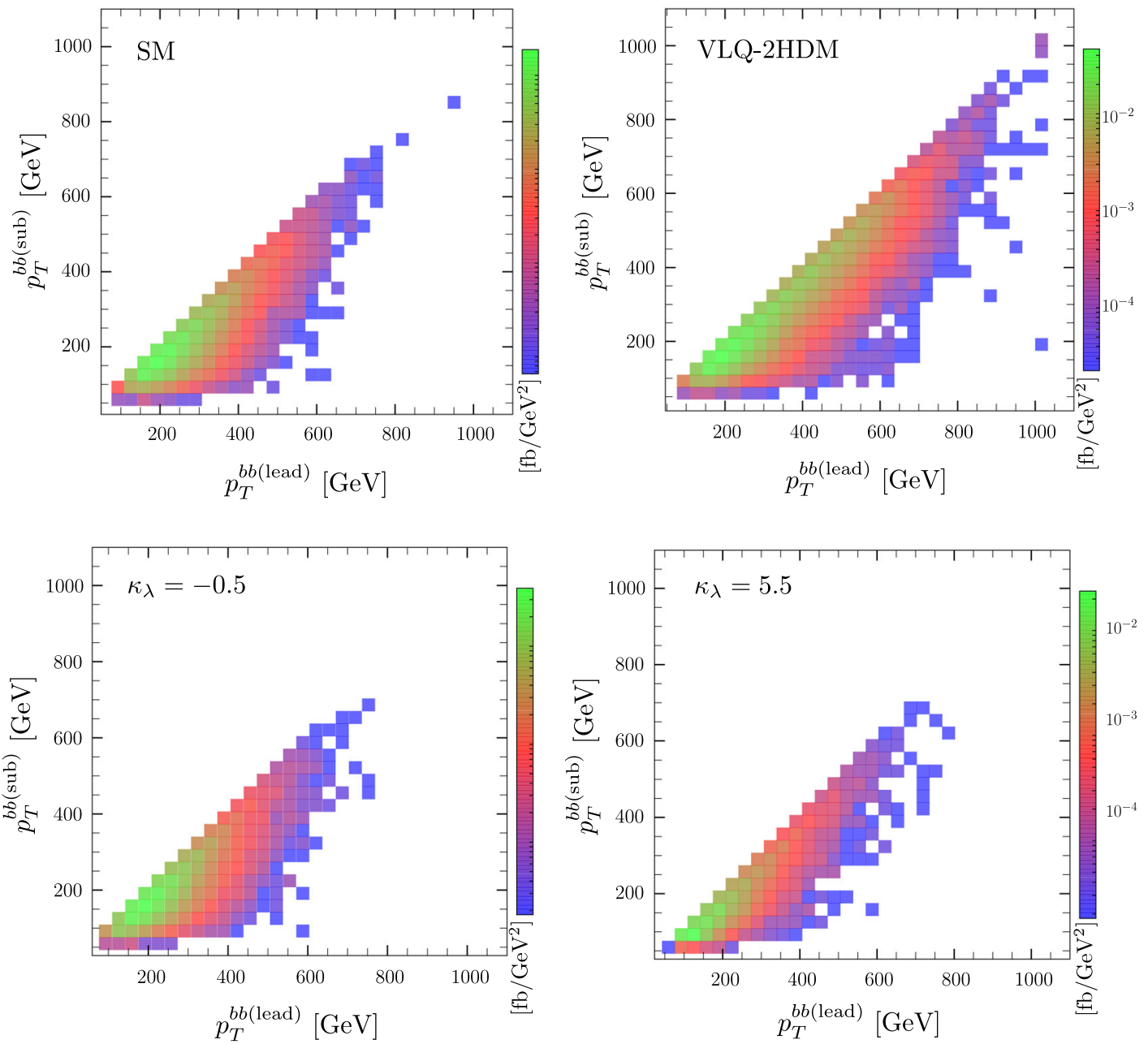

FIG. 6. $d^{2} \sigma / d p_{T}^{b b(\text { lead) }} / d p_{T}^{b b(\text { sub) }}$ in units of fb/ $/ \mathrm{GeV}^{2}$, where $p_{T}^{b b(\text { lead) }}$ is the transverse momentum of the leading dijet and $p_{T}^{b b(\text { sub) }}$ is that of the subleading dijet, in the SM (upper left), the VLQ-2HDM (upper right), the SM with $\kappa_{\lambda}=-0.5$ (lower left) and $\kappa_{\lambda}=5.5$ (lower right). 
one dijet system. This selection step reduces the number of events in the SM by a factor of about 2 .

In Fig. 5, we show the distributions of the transverse momentum of the leading dijet $p_{T}^{b b \text { (lead) }}$ (left panel) and the invariant mass of the $4 b$ system for $g g \rightarrow h h \rightarrow 4 b$ in the VLQ-2HDM (black), the SM (red), the SM with $\kappa_{\lambda}=-0.5$ (blue), and $\kappa_{\lambda}=5.5$ (green). We first remark that in the $\kappa_{\lambda}=5.5$ case, the total number of events (originally corresponding to $\left.\sigma_{\mathrm{NP}} / \sigma_{\mathrm{SM}}(g g \rightarrow h h) \simeq 3\right)$ is considerably reduced and the peaks of both distributions are shifted toward low values. This is because some $b$-jets in the event are too soft to pass the first selection $p_{T}^{b}>40 \mathrm{GeV}$ [68]. An encouraging observation is that the threshold effects of the VLQs are visible at the reconstruction level. We can see two bumplike structures in both $p_{T}^{b b(\text { lead })}$ and $M_{4 b}$ distributions, peaked at $p_{T}^{b b(\text { lead })} \sim M_{1}$ and $M_{4 b} \sim 2 M_{1}$, with a minor smearing effect due to the detector angularity. Since the two peak positions are closely related, a study of the correlation between the two observables will be extremely useful to probe new VLQs in the di-Higgs process.
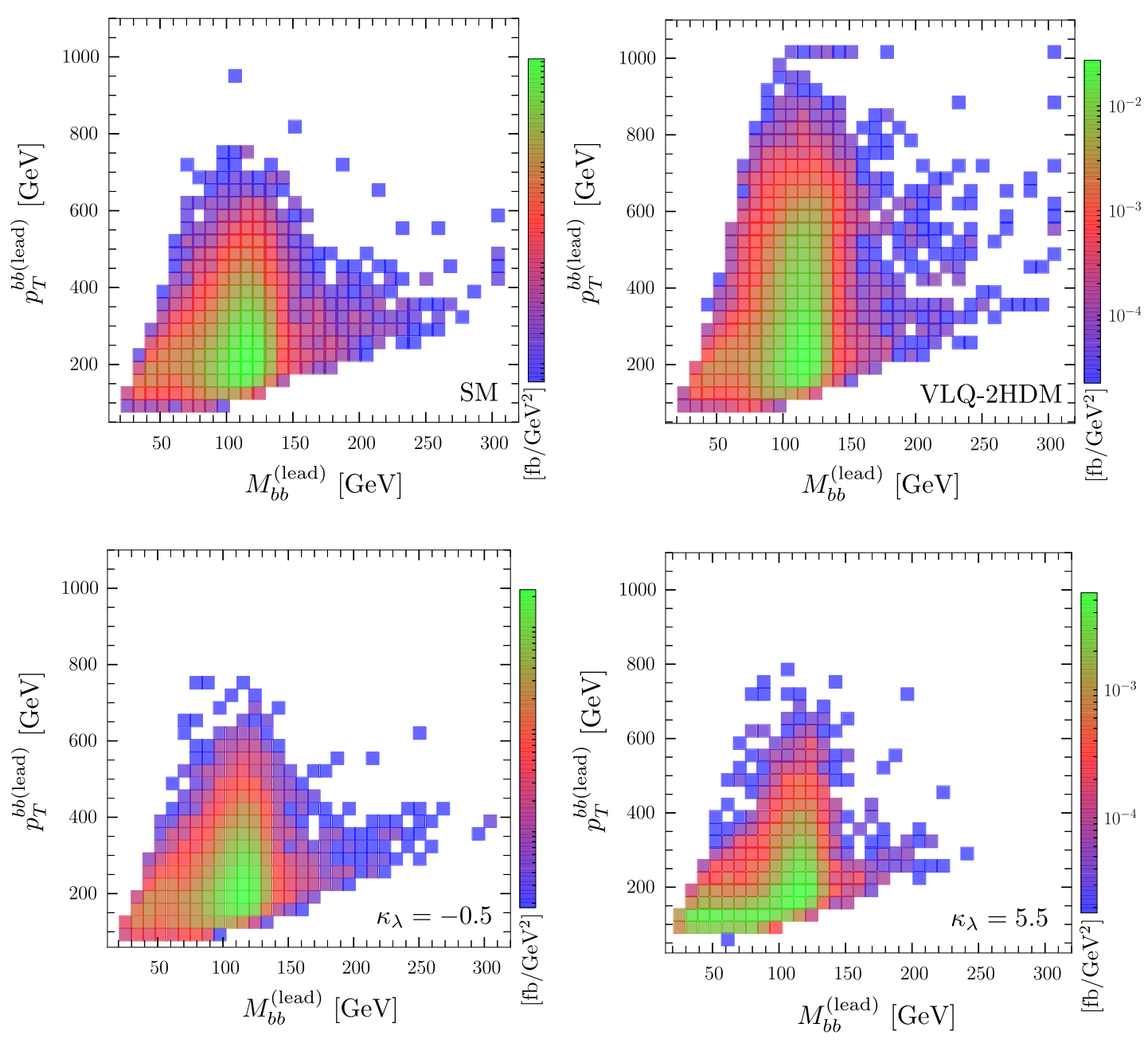

FIG. 7. Same as Fig. 6 but for $d^{2} \sigma / d M_{b b}^{\text {(lead) }} / d p_{T}^{b b(\text { lead })}$.

Motivated by the correlated bumps in the $p_{T}^{b b(\text { lead })}$ and $M_{4 b}$ distributions, we study the double differential cross sections in some key variables. In Fig. 6, we show one as a function of the transverse momentum of the leading dijet and the transverse momentum of the sub-leading dijet, $d^{2} \sigma / d p_{T}^{b b(\text { lead) }} / d p_{T}^{b b(\text { sub) }}$, in units of $\mathrm{fb} / G \mathrm{eV}^{2}$. We consider the SM (upper left), the VLQ-2HDM (upper right), the SM with $\kappa_{\lambda}=-0.5$ (lower left) and $\kappa_{\lambda}=5.5$ (lower right). The generic correlation of $p_{T}^{b b \text { (lead) }} \simeq p_{T}^{b b \text { (sub) }}$, originated from the back-to-back motion of two Higgs bosons, is common for all four models. The main difference is the kinematic area corresponding to sizable $d^{2} \sigma / d p_{T}^{b b \text { (lead) } / ~}$ $d p_{T}^{b b(\mathrm{sub})}$, which is the largest for the VLQ-2HDM and the smallest for the case of $\kappa_{\lambda}=5.5$. In the region of $p_{T}^{b b \text { (lead,sub) }}>400 \mathrm{GeV}$, only the VLQ-2HDM accommodates $d^{2} \sigma / d p_{T}^{b b(\text { lead })} / d p_{T}^{b b \text { (sub) }} \gtrsim 10^{-2} \mathrm{fb} / \mathrm{GeV}^{2}$. This unique feature is very useful for discriminating the VLQ-2HDM.

In Fig. 7, we display the double differential cross section in the invariant mass and transverse momentum of the leading dijet for the four models as shown in Fig. 6. 

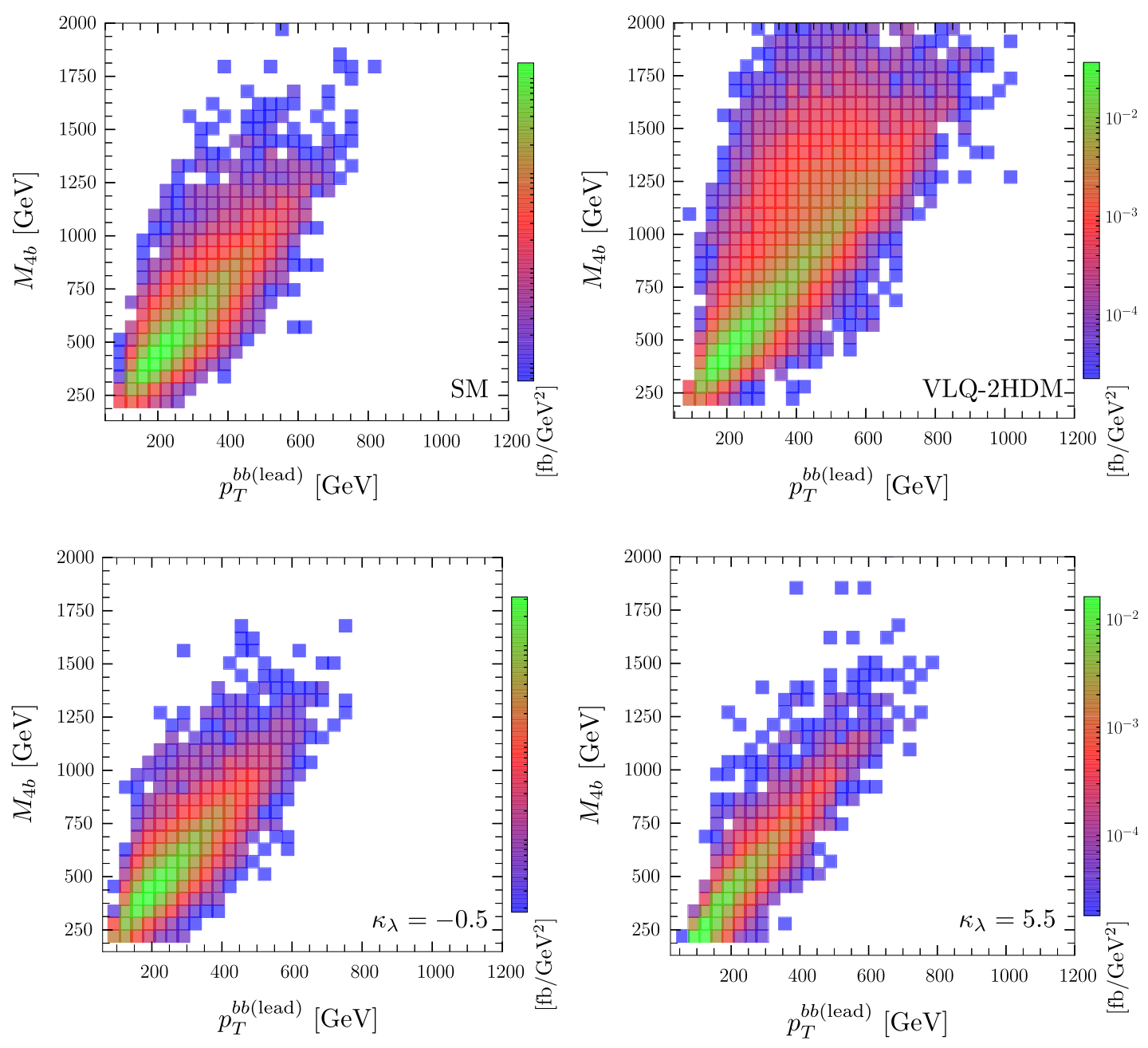

FIG. 8. Same as Fig. 6 but for $d^{2} \sigma / d p_{T}^{b b(\text { lead })} / d M_{4 b}$.

The distributions are well localized around the SM Higgs boson mass window $\left(M_{b b}^{(\text {lead })} \simeq m_{h}\right)$ in all of the models except for the case $\kappa_{\lambda}=5.5$ where a sizable number of events yield $M_{b b}^{\text {(lead) }} \lesssim m_{h}$. The LHC discovery prospect for $\kappa_{\lambda}=5.5$ is expected to be low, because the usual $m_{h}$ window cut removes a considerable part of the $\kappa_{\lambda}=5.5$ signal. Figure 7 also shows that the correlation between $M_{b b}^{\text {(lead) }}$ and $p_{T}^{b b(\text { lead })}$ is very weak in all of the four models. Therefore, selecting events with high transverse momentum for the leading (or the subleading) dijet does not alter the requirement on the Higgs boson mass windows. ${ }^{10}$ For the subleading dijet, we find that the double differential cross section about its invariant mass and its transverse momentum shows a similar behavior as in Fig. 7.

\footnotetext{
${ }^{10}$ Due to the different dynamics of the $4 b$ final state from other decay modes of the di-Higgs process, the signal region requires $X_{h h}<1.6$ where $X_{h h}=\sqrt{\left(\frac{M_{b b}^{\text {(lead) }}-124 \mathrm{GeV}}{0.1 M_{b b}^{(\text {lead) }}}\right)^{2}+\left(\frac{M_{b b}^{\text {(sub) }}-115 \mathrm{GeV}}{0.1 M_{b b}^{\text {(sub) }}}\right)^{2}}$.
} Here a resolution of $10 \%$ on the mass of the two dijets is assumed.
Targeting two correlated bumps around $p_{T}^{h} \simeq M_{1}$ and $M_{h h} \simeq 2 M_{1}$ in the VLQ-2HDM, we present the double differential cross section $d^{2} \sigma / d p_{T}^{b b \text { (lead) }} / d M_{4 b}$ in Fig. 8. We observe a strong correlation along the line of $M_{4 b} \simeq$ $2 p_{T}^{b b \text { (lead) }}$ in all of the four models. The unique feature of the VLQ-2HDM is the extent of the correlation line with substantial $d^{2} \sigma / d p_{T}^{h} / d M_{h h}$. Selecting the events along the

TABLE II. Sequence of the event preselection in $h h \rightarrow b \bar{b} \gamma \gamma$ channel at the HL-LHC.

\begin{tabular}{ll}
\hline \hline Sequence & Event Preselection at the HL-LHC \\
\hline 1 & $\begin{aligned} \text { Di-photon trigger condition: } \geq 2 \text { isolated photons } \\
\quad \text { with } p_{T}^{\gamma}>25 \mathrm{GeV} \text { and }\left|\eta_{\gamma}\right|<2.5\end{aligned}$ \\
2 & 2 isolated photons with $p_{T}^{\gamma}>30 \mathrm{GeV},\left|\eta_{\gamma}\right|<$ \\
& 1.37 or $1.52<\left|\eta_{\gamma}\right|<2.37$, and $\Delta R_{j \gamma}>0.4$ \\
3 & $2 b$-jets with leading (sub-leading) $p_{T}^{b}>$ \\
& $40(30) \mathrm{GeV}$ and $|\eta|<2.4$ \\
4 & $0.4<\Delta R_{b b}<2.0$ and $0.4<\Delta R_{\gamma \gamma}<2.0$ \\
\hline \hline
\end{tabular}


TABLE III. Cut flow efficiencies of four models for the diHiggs process in the $b \bar{b} \gamma \gamma$ final state at the HL-LHC.

\begin{tabular}{lrrrr}
\hline \hline Sequence & SM & VLQ-2HDM & $\kappa_{\lambda}=-0.5$ & $\kappa_{\lambda}=5.5$ \\
\hline 1 & $27.60 \%$ & $29.71 \%$ & $25.19 \%$ & $20.46 \%$ \\
2 & $25.47 \%$ & $25.88 \%$ & $23.02 \%$ & $18.12 \%$ \\
3 & $19.31 \%$ & $18.35 \%$ & $17.27 \%$ & $12.86 \%$ \\
4 & $5.43 \%$ & $4.78 \%$ & $4.14 \%$ & $1.51 \%$ \\
\hline \hline
\end{tabular}

line of $M_{4 b} \simeq 2 p_{T}^{b b(\text { lead })}$ in high $M_{h h}$ region will be one of the most sensitive probes for the VLQ-2HDM effects on the di-Higgs process.

\section{B. $b \bar{b} \gamma \gamma$ final state}

For the analysis of the $b \bar{b} \gamma \gamma$ final state of the di-Higgs process, we follow the ATLAS reports $[149,150]$. For the photon identification efficiency $\epsilon_{\gamma}$, we fit to the ATLAS simulation results and obtain the following dependence of $\epsilon_{\gamma}$ on the photon transverse momentum $p_{T}^{\gamma}$ :

$$
\epsilon_{\gamma}=0.888 * \tanh \left(0.01275 \frac{p_{T}^{\gamma}}{\mathrm{GeV}}\right) .
$$

The probabilities for a jet and an electron to fake a photon, the photon fake rates, are $P_{j \rightarrow \gamma}=5 \times 10^{-4}$ and $P_{e \rightarrow \gamma}=$ $2 \%(5 \%)$ in the barrel (endcap) region [149]. For the $b$ tagging efficiency, we have adopted the dependence of $\epsilon_{b}$ on the transverse momentum and rapidity of the $b$-jet in Fig. 7(b) of Ref. [151]. The misidentification probability of the charm quark jet as the $b$-jet, $P_{c \rightarrow b}$, depends not only on the $b$-tagging efficiency but also the transverse momentum and rapidity of the $c$-jet. The $\epsilon_{b}$ dependence is incorporated by taking the multi-variate MV1 $b$-tagging algorithm with $P_{c \rightarrow b} \simeq 1 / 5$ for $\epsilon_{b}=0.7$ and $P_{c \rightarrow b} \simeq 1$ as $\epsilon_{b} \rightarrow 1$ [152]. The dependence of $P_{c \rightarrow b}$ on $p_{T}^{c}$ and $\eta^{c}$ is also included. For the light-jet fake rate as the $b$ jet, we take $P_{j \rightarrow b}=1 / 1300$
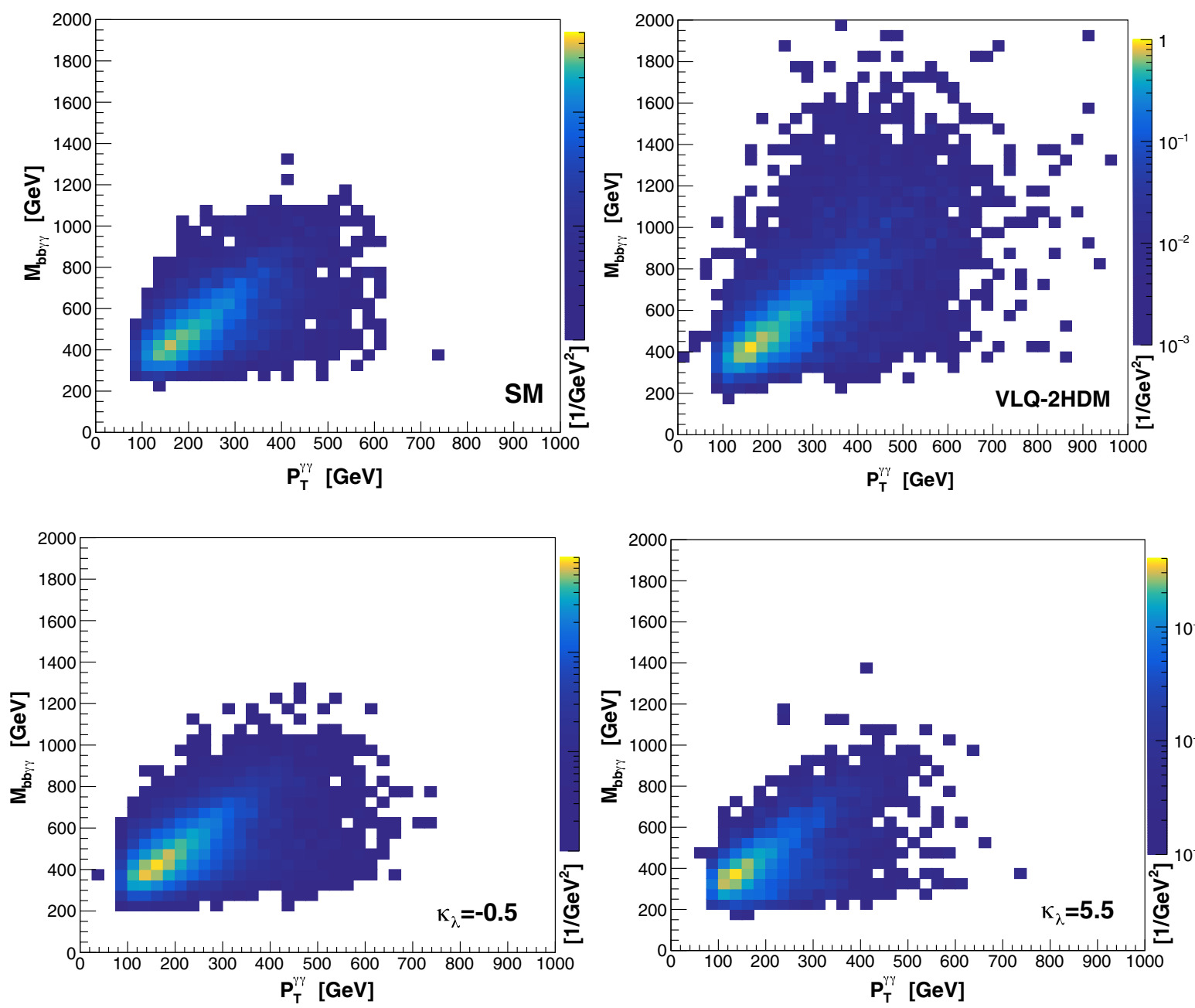

FIG. 9. The distribution of the number of events versus the transverse momentum of the di-photon and the invariant mass of $b \bar{b} \gamma \gamma$ for the $b \bar{b} \gamma \gamma$ final state of the di-Higgs process at the HL-LHC. We consider the SM (upper left), the VLQ-2HDM (upper right), the SM with $\kappa_{\lambda}=-0.5$ (lower left), and $\kappa_{\lambda}=5.5$ (lower right). 
[149]. The pile-up effects are not considered, based on the reasonings in Ref. [68]. The last consideration for a realistic analysis is the energy loss in the $b$ momentum reconstruction, which is taken into account by the jetenergy scaling factor of

$$
X_{E_{b}}=\sqrt{\frac{\left(3.0-0.2\left|\eta_{b}\right|\right)^{2}}{p_{T}^{b} / \mathrm{GeV}}+1.27},
$$

where the factor 1.27 is obtained by requiring a correct peak position at $M_{b b}=m_{h}$.

Referring to the ATLAS di-Higgs study in Ref. [149], we take a sequence of the event preselection for $g g \rightarrow h h \rightarrow b \bar{b} \gamma \gamma$ in Table II. We found that other preselections in Ref. [149] are not useful for our signal. In Table III, we show the efficiencies of each sequence in four different models. The efficiencies are similar for the SM, the VLQ-2HDM, and the SM with $\kappa_{\lambda}=-0.5$, being about $4-5 \%$ at the final step. In the case $\kappa_{\lambda}=5.5$, however, the efficiency dramatically drops after the Selection-4, about a third of that in the other three models. This is because the most events for the case $\kappa_{\lambda}=5.5$ are with $\Delta R_{\gamma \gamma}>2.0$ region like the main SM backgrounds [68]. Even considering $\sigma_{\mathrm{NP}} / \sigma_{\mathrm{SM}} \simeq 3$ for $\kappa_{\lambda}=5.5, \sigma \times \mathcal{B}$ is about $80 \%$ of the $\mathrm{SM}$ result after the Selection-4. It is very challenging to probe at the HL-LHC.

In Fig. 9, we present $d^{2} N / d p_{T}^{\gamma \gamma} / d M_{b \bar{b} \gamma \gamma}$, the distribution of the number of events versus the transverse momentum of the diphoton and the invariant mass of $b \bar{b} \gamma \gamma$, in the SM (upper left), the VLQ-2HDM (upper right), the SM with $\kappa_{\lambda}=-0.5$ (lower left), and $\kappa_{\lambda}=5.5$ (lower right). Since the $b \bar{b} \gamma \gamma$ final state has an extremely small cross section, we show the distribution of the number of events corresponding to the total integrated luminosity of $3000 \mathrm{fb}^{-1}$. The overall characteristics are very similar to those in the $b \bar{b} b \bar{b}$ final state: there is a strong correlation along the line $M_{b \bar{b} \gamma \gamma} \simeq 2 p_{T}^{\gamma \gamma}$ in all of the four models; the VLQ-2HDM yields the widest spread up to high $p_{T}^{\gamma \gamma}$ and $M_{b \bar{b} \gamma \gamma}$; the $\kappa_{\lambda}=$ 5.5 case prefers small $p_{T}^{\gamma \gamma}$ and $M_{b \bar{b} \gamma \gamma}$, compared with the other models. If we count the bins with $d^{2} N / d p_{T}^{\gamma \gamma} /$ $d M_{b \bar{b} \gamma \gamma}>1 / \mathrm{GeV}^{2}$, however, it is very difficult to see the difference among different NP models. Moreover, the isolation condition, $\Delta R_{\gamma \gamma}, \Delta R_{b b}>0.4$, also restricts the power to detect high $p_{T}^{\gamma \gamma}, p_{T}^{b \bar{b}}$ regions in the $b \bar{b} \gamma \gamma$ final state. In summary, the $b \bar{b} \gamma \gamma$ final state plays a complementary role in observing the di-Higgs process, but not appropriate for the deeper study of NP.

\section{CONCLUSIONS}

With the aim of disentangling different NP contributions to the di-Higgs process from gluon fusion, we have studied the phenomenological characteristics of the kinematical distributions, focusing on the double differential cross sections. For illustration purposes, we assume that the NP effects would first appear in the total production cross section, being three times as large as the SM expectation. Since we can easily identify resonant di-Higgs production, we concentrated on the nonresonant NP effects from nonSM Higgs trilinear couplings $\left(\kappa_{\lambda}=-0.5\right.$ or $\left.\kappa_{\lambda}=5.5\right)$ and the new colored fermions running in the loop. For the latter, we need a concrete NP model for a comprehensive study since new quarks, which enhance the di-Higgs production rate, should similarly act in the single-Higgs production.

In this work, we have studied a type-II 2HDM with vectorlike quarks, called the VLQ-2HDM. The electroweak oblique parameters remain almost the same as in the SM by adopting an ansatz that guarantees a vanishing $\hat{T}$, called the zero- $\hat{T}$ ansatz: see Eq. (17). We analytically calculated the new form factors from the VLQs. In order to show the role of the Higgs-fermion-fermion couplings in breaking the correlation between the di-Higgs and single-Higgs processes, we considered the alignment limit and the exact wrong-sign (EWS) limit. In the alignment limit, both uptype and down-type VLQs have the same-sign couplings to the Higgs boson, so that their contributions to the triangle diagrams of the di-Higgs process are constructive to each other. Moreover they are strongly correlated with the VLQ contributions to the box diagrams. As the single-Higgs process has the same triangle diagrams, we cannot accommodate $\sigma_{\mathrm{NP}} / \sigma_{\mathrm{SM}}(g g \rightarrow h h) \simeq 3$ and $\sigma_{\mathrm{NP}} / \sigma_{\mathrm{SM}}(g g \rightarrow h) \simeq 1$ simultaneously: the maximum increase of the di-Higgs production rate allowed by the observed $\kappa_{g}$ is only $20 \%$. In the EWS limit, however, the down-type and up-type VLQs have opposite sign Yukawa couplings, thus yielding a considerable cancellation between their contributions to single-Higgs production. The box diagrams do not have this kind of cancellation because their amplitudes are proportional to the square of the Higgs-fermion-fermion coupling. Significant enhancement of the total production cross section of the di-Higgs process is feasible in the EWS limit, where we took a benchmark point.

First at parton level, we calculated the kinematic distributions for the $g g \rightarrow h h$ process in three NP models, $\kappa_{\lambda}=-0.5, \kappa_{\lambda}=5.5$, and the VLQ-2HDM. Although they have almost the same total production cross section of $\sigma_{\mathrm{NP}} / \sigma_{\mathrm{SM}}(g g \rightarrow h h) \simeq 3$, the $M_{h h}$ and $p_{T}^{h}$ distributions show quite significant differences. The $\kappa_{\lambda}=-0.5$ model yields similar distribution shapes to the SM results. In the $\kappa_{\lambda}=5.5$ model, both distributions apparently shift toward low $M_{h h}$ or $p_{T}^{h}$ region such that the peak position moves about $100 \mathrm{GeV}$. This feature makes the $\kappa_{\lambda}=5.5$ model very challenging to probe at the LHC, because the SM backgrounds to the di-Higgs process such as $4 b, b \bar{b} c \bar{c}$, and $t \bar{t}$ are populated in the low $p_{T}^{h}$ region. The VLQ-2HDM showed its unique and distinctive features in the $M_{h h}$ and $p_{T}^{h}$ distributions, benefitting from the VLQ threshold effects. At parton level, we could clearly see the bumps around $M_{h h} \simeq 2 M_{1}$ and $p_{T}^{h} \simeq M_{1}$, where $M_{1}$ is the lightest VLQ 
mass. Moreover, the bumps of the threshold origin from heavy VLQs naturally lift up the kinematic distributions of $M_{h h}$ and $p_{T}^{h}$ into high regions. The doubly high region, with high $M_{h h}$ and high $p_{T}^{h}$, can be the exclusive territory of the VLQ-2HDM for the di-Higgs process.

We also have completed the analysis with full collider simulations for the di-Higgs signals in the VLQ-2HDM, the $\mathrm{SM}$, the SM with $\kappa_{\lambda}=-0.5$, and with $\kappa_{\lambda}=5.5$. Two final states, $b \bar{b} b \bar{b}$ and $b \bar{b} \gamma \gamma$ of the decays of the Higgs-boson pair, were studied. Fortunately, many characteristic features at the parton-level calculation survived even after parton showering, hadronization, and detector simulations. The bump structures in the distributions of $M_{h h}$ and $p_{T}^{h}$, though being smeared a little bit, are maintained, and the positions of the peaks roughly stay at the same place. Motivated by the correlation of the bumps in $M_{h h}$ and $p_{T}^{h}$ distributions, we studied various double differential cross sections. In the $b \bar{b} b \bar{b}$ final state, we first found that any selection on the transverse momentum of the leading (or the sub-leading) dijets since a Higgs boson candidate barely alters its invariant mass. The smoking-gun signature appears in $d^{2} \sigma / d M_{h h} / d p_{T}^{h}$. All four models showed a strong correlation along the line of $M_{h h} \simeq 2 p_{T}^{h}$, which is also useful to search for the SM di-Higgs process itself. Distinguishing the VLQ-2HDM from other NP models is possible in the $b \bar{b} b \bar{b}$ final state as the observable correlation line of $M_{h h} \simeq 2 p_{T}^{h}$ is the longest, extending far toward high $p_{T}^{h}$ region: the case $\kappa_{\lambda}=5.5$ has the shortest. However, the $b \bar{b} \gamma \gamma$ final state has too small signal rate, not appropriate to see the difference among the NP models. In summary, we expect that our observation of the correlation between $M_{h h}$ and $p_{T}^{h}$ distributions for disentangling the NP effects on the di-Higgs process can help the NP search at the HL-LHC.

\section{ACKNOWLEDGMENTS}

K. C. was supported by the MoST of Taiwan under Grant No. MOST-107-2112-M-007-029-MY3. The work of A. J. and J. S. is supported by the National Research Foundation of Korea, Grant No. NRF-2019R1A2C1009419. The work of Y.W. Y. was supported by the National Research Foundation of Korea grant funded by the Korea government (No. 2019R1I1A1A01064113).
[1] G. Aad et al. (ATLAS Collaboration), Observation of a new particle in the search for the Standard Model Higgs boson with the ATLAS detector at the LHC, Phys. Lett. B 716, 1 (2012).

[2] S. Chatrchyan et al. (CMS Collaboration), Observation of a new boson at a mass of $125 \mathrm{GeV}$ with the CMS experiment at the LHC, Phys. Lett. B 716, 30 (2012).

[3] G. Aad et al. (ATLAS Collaboration), Combined measurements of Higgs boson production and decay using up to $80 \mathrm{fb}^{-1}$ of proton-proton collision data at $\sqrt{s}=13 \mathrm{TeV}$ collected with the ATLAS experiment, Phys. Rev. D 101, 012002 (2020).

[4] CMS Collaboration, Sensitivity projections for Higgs boson properties measurements at the HL-LHC, CERN Report No. CMS-PAS-FTR-18-011, 2018.

[5] J. de Blas et al., Higgs boson studies at future particle colliders, J. High Energy Phys. 01 (2020) 139.

[6] T. Plehn and M. Rauch, The quartic Higgs coupling at hadron colliders, Phys. Rev. D 72, 053008 (2005).

[7] G. Li, L.-X. Xu, B. Yan, and C. P. Yuan, Resolving the degeneracy in top quark Yukawa coupling with Higgs pair production, Phys. Lett. B 800, 135070 (2020).

[8] Q.-H. Cao, G. Li, B. Yan, D.-M. Zhang, and H. Zhang, Double Higgs production at the $14 \mathrm{TeV}$ LHC and a $100 \mathrm{TeV} p p$ collider, Phys. Rev. D 96, 095031 (2017).

[9] Q.-H. Cao, B. Yan, D.-M. Zhang, and H. Zhang, Resolving the degeneracy in single Higgs production with Higgs pair production, Phys. Lett. B 752, 285 (2016).
[10] E. W. N. Glover and J. J. van der Bij, Higgs boson pair production via gluon fusion, Nucl. Phys. B309, 282 (1988).

[11] T. Plehn, M. Spira, and P. M. Zerwas, Pair production of neutral Higgs particles in gluon-gluon collisions, Nucl. Phys. B479, 46 (1996); , Erratum, Nucl. Phys. B531, 655 (1998).

[12] A. Djouadi, W. Kilian, M. Muhlleitner, and P. M. Zerwas, Production of neutral Higgs boson pairs at LHC, Eur. Phys. J. C 10, 45 (1999).

[13] M. J. Dolan, C. Englert, and M. Spannowsky, New physics in LHC Higgs boson pair production, Phys. Rev. D 87, 055002 (2013).

[14] C.-Y. Chen, S. Dawson, and I. M. Lewis, Exploring resonant di-Higgs boson production in the Higgs singlet model, Phys. Rev. D 91, 035015 (2015).

[15] J. M. No and M. Ramsey-Musolf, Probing the Higgs portal at the LHC through resonant di-Higgs production, Phys. Rev. D 89, 095031 (2014).

[16] A. Alves, T. Ghosh, H.-K. Guo, and K. Sinha, Resonant diHiggs production at gravitational wave benchmarks: A collider study using machine learning, J. High Energy Phys. 12 (2018) 070.

[17] A. Adhikary, S. Banerjee, R. K. Barman, B. Bhattacherjee, and S. Niyogi, Revisiting the non-resonant Higgs pair production at the HL-LHC, J. High Energy Phys. 07 (2018) 116.

[18] A. Alves, D. Gonalves, T. Ghosh, H.-K. Guo, and K. Sinha, Di-Higgs production in the $4 b$ channel and 
gravitational wave complementarity, J. High Energy Phys. 03 (2020) 053.

[19] E. Asakawa, D. Harada, S. Kanemura, Y. Okada, and K. Tsumura, Higgs boson pair production in new physics models at hadron, lepton, and photon colliders, Phys. Rev. D 82, 115002 (2010).

[20] J. Baglio, A. Djouadi, R. Grber, M. M. Mhlleitner, J. Quevillon, and M. Spira, The measurement of the Higgs self-coupling at the LHC: Theoretical status, J. High Energy Phys. 04 (2013) 151.

[21] V. Barger, L. L. Everett, C. B. Jackson, and G. Shaughnessy, Higgs-pair production and measurement of the triscalar coupling at $\operatorname{LHC}(8,14)$, Phys. Lett. B 728, 433 (2014).

[22] M. Slawinska, W. van den Wollenberg, B. van Eijk, and S. Bentvelsen, Phenomenology of the trilinear Higgs coupling at proton-proton colliders, arXiv:1408.5010.

[23] B. Batell, M. McCullough, D. Stolarski, and C. B. Verhaaren, Putting a stop to di-Higgs modifications, J. High Energy Phys. 09 (2015) 216.

[24] P. Huang, A. Joglekar, M. Li, and C. E. M. Wagner, Corrections to di-Higgs boson production with light stops and modified Higgs couplings, Phys. Rev. D 97, 075001 (2018).

[25] G. D. Kribs and A. Martin, Enhanced di-Higgs production through light colored scalars, Phys. Rev. D 86, 095023 (2012).

[26] S. Dawson, A. Ismail, and I. Low, What's in the loop? The anatomy of double Higgs production, Phys. Rev. D 91, 115008 (2015).

[27] J. Cao, Z. Heng, L. Shang, P. Wan, and J. M. Yang, Pair production of a $125 \mathrm{GeV}$ Higgs boson in MSSM and NMSSM at the LHC, J. High Energy Phys. 04 (2013) 134.

[28] C. Han, X. Ji, L. Wu, P. Wu, and J. M. Yang, Higgs pair production with SUSY QCD correction: Revisited under current experimental constraints, J. High Energy Phys. 04 (2014) 003.

[29] H.-J. He, J. Ren, and W. Yao, Probing new physics of cubic Higgs boson interaction via Higgs pair production at hadron colliders, Phys. Rev. D 93, 015003 (2016).

[30] A. Alloul, B. Fuks, and V. Sanz, Phenomenology of the Higgs effective Lagrangian via FEYNRULES, J. High Energy Phys. 04 (2014) 110.

[31] A. Pierce, J. Thaler, and L.-T. Wang, Disentangling dimension six operators through di-Higgs boson production, J. High Energy Phys. 05 (2007) 070.

[32] G. Buchalla, M. Capozi, A. Celis, G. Heinrich, and L. Scyboz, Higgs boson pair production in non-linear effective field theory with full $m_{t}$-dependence at NLO QCD, J. High Energy Phys. 09 (2018) 057.

[33] M. Bauer, M. Carena, and A. Carmona, Higgs Pair Production as a Signal of Enhanced Yukawa Couplings, Phys. Rev. Lett. 121, 021801 (2018).

[34] L. Alasfar, R. Corral Lopez, and R. Grber, Probing Higgs couplings to light quarks via Higgs pair production, J. High Energy Phys. 11 (2019) 088.

[35] G. Aad et al. (ATLAS Collaboration), Combination of searches for Higgs boson pairs in $p p$ collisions at $\sqrt{s}=$ $13 \mathrm{TeV}$ with the ATLAS detector, Phys. Lett. B 800, 135103 (2020).
[36] A. M. Sirunyan et al. (CMS Collaboration), Combination of Searches for Higgs Boson Pair Production in Proton-Proton Collisions at $\sqrt{s}=13 \mathrm{TeV}$, Phys. Rev. Lett. 122, 121803 (2019).

[37] F. Kling, T. Plehn, and P. Schichtel, Maximizing the significance in Higgs boson pair analyses, Phys. Rev. D 95, 035026 (2017).

[38] D. Gonalves, T. Han, F. Kling, T. Plehn, and M. Takeuchi, Higgs boson pair production at future hadron colliders: From kinematics to dynamics, Phys. Rev. D 97, 113004 (2018).

[39] U. Baur, T. Plehn, and D. L. Rainwater, Measuring the Higgs Boson Self Coupling at the LHC and Finite Top Mass Matrix Elements, Phys. Rev. Lett. 89, 151801 (2002).

[40] U. Baur, T. Plehn, and D. L. Rainwater, Determining the Higgs boson self-coupling at hadron colliders, Phys. Rev. D 67, 033003 (2003).

[41] R. Grober, M. Muhlleitner, and M. Spira, Signs of composite Higgs pair production at next-to-leading order, J. High Energy Phys. 06 (2016) 080.

[42] S. Dawson, E. Furlan, and I. Lewis, Unravelling an extended quark sector through multiple Higgs production?, Phys. Rev. D 87, 014007 (2013).

[43] G. C. Branco, P. M. Ferreira, L. Lavoura, M. N. Rebelo, M. Sher, and J. P. Silva, Theory and phenomenology of two-Higgs-doublet models, Phys. Rep. 516, 1 (2012).

[44] P. M. Ferreira, J. F. Gunion, H. E. Haber, and R. Santos, Probing wrong-sign Yukawa couplings at the LHC and a future linear collider, Phys. Rev. D 89, 115003 (2014).

[45] P. M. Ferreira, R. Guedes, M. O. P. Sampaio, and R. Santos, Wrong sign and symmetric limits and nondecoupling in 2HDMs, J. High Energy Phys. 12 (2014) 067.

[46] A. Biswas and A. Lahiri, Alignment, reverse alignment, and wrong sign Yukawa couplings in two Higgs doublet models, Phys. Rev. D 93, 115017 (2016).

[47] S. K. Kang, Z. Qian, J. Song, and Y. W. Yoon, Confronting the fourth generation two Higgs doublet model with the phenomenology of heavy Higgs bosons, Phys. Rev. D 98, 095025 (2018).

[48] D. Das, A. Kundu, and I. Saha, Higgs data does not rule out a sequential fourth generation with an extended scalar sector, Phys. Rev. D 97, 011701 (2018).

[49] G. Cacciapaglia, A. Deandrea, L. Panizzi, N. Gaur, D. Harada, and Y. Okada, Heavy vector-like top partners at the LHC and flavour constraints, J. High Energy Phys. 03 (2012) 070.

[50] J. A. Aguilar-Saavedra, R. Benbrik, S. Heinemeyer, and M. Prez-Victoria, Handbook of vectorlike quarks: Mixing and single production, Phys. Rev. D 88, 094010 (2013).

[51] S. A. R. Ellis, R. M. Godbole, S. Gopalakrishna, and J. D. Wells, Survey of vector-like fermion extensions of the standard model and their phenomenological implications, J. High Energy Phys. 09 (2014) 130.

[52] A. Angelescu, A. Djouadi, and G. Moreau, Scenarii for interpretations of the LHC diphoton excess: Two Higgs doublets and vector-like quarks and leptons, Phys. Lett. B 756, 126 (2016). 
[53] A. Arhrib, R. Benbrik, S. J. D. King, B. Manaut, S. Moretti, and C.S. Un, Phenomenology of 2HDM with vectorlike quarks, Phys. Rev. D 97, 095015 (2018).

[54] D. Barducci and L. Panizzi, Vector-like quarks coupling discrimination at the LHC and future hadron colliders, J. High Energy Phys. 12 (2017) 057.

[55] G. Cacciapaglia, H. Cai, A. Carvalho, A. Deandrea, T. Flacke, B. Fuks, D. Majumder, and H.-S. Shao, Probing vector-like quark models with Higgs-boson pair production, J. High Energy Phys. 07 (2017) 005.

[56] A. Arhrib, R. Benbrik, J. El Falaki, M. Sampaio, and R. Santos, Pseudoscalar decays to gauge bosons at the LHC and at a future $100 \mathrm{TeV}$ collider, Phys. Rev. D 99, 035043 (2019).

[57] G. Cacciapaglia, A. Carvalho, A. Deandrea, T. Flacke, B. Fuks, D. Majumder, L. Panizzi, and H.-S. Shao, Next-toleading-order predictions for single vector-like quark production at the LHC, Phys. Lett. B 793, 206 (2019).

[58] J. Song and Y.W. Yoon, $W \gamma$ decay of the elusive charged Higgs boson in the two-Higgs-doublet model with vectorlike fermions, Phys. Rev. D 100, 055006 (2019).

[59] L. Randall and R. Sundrum, A Large Mass Hierarchy from a Small Extra Dimension, Phys. Rev. Lett. 83, 3370 (1999).

[60] H.-C. Cheng, B. A. Dobrescu, and C. T. Hill, Electroweak symmetry breaking and extra dimensions, Nucl. Phys. B589, 249 (2000).

[61] N. Arkani-Hamed, A. G. Cohen, E. Katz, and A. E. Nelson, The littlest Higgs, J. High Energy Phys. 07 (2002) 034.

[62] T. Han, H. E. Logan, B. McElrath, and L.-T. Wang, Loop induced decays of the little Higgs: H->gg, gamma gamma, Phys. Lett. B 563, 191 (2003); , Erratum, Phys. Lett. B 603, 257 (2004).

[63] H.-C. Cheng, I. Low, and L.-T. Wang, Top partners in little Higgs theories with T-parity, Phys. Rev. D 74, 055001 (2006).

[64] J. Kang, P. Langacker, and B. D. Nelson, Theory and phenomenology of exotic isosinglet quarks and squarks, Phys. Rev. D 77, 035003 (2008).

[65] C. Anastasiou, S. Buehler, E. Furlan, F. Herzog, and A. Lazopoulos, Higgs production cross-section in a standard model with four generations at the LHC, Phys. Lett. B 702, 224 (2011).

[66] C. Anastasiou, C. Duhr, F. Dulat, E. Furlan, T. Gehrmann, F. Herzog, A. Lazopoulos, and B. Mistlberger, High precision determination of the gluon fusion Higgs boson cross-section at the LHC, J. High Energy Phys. 05 (2016) 058.

[67] M.E. Peskin and T. Takeuchi, Estimation of oblique electroweak corrections, Phys. Rev. D 46, 381 (1992).

[68] J. Chang, K. Cheung, J.S. Lee, C.-T. Lu, and J. Park, Higgs-boson-pair production $h(\rightarrow b \bar{b}) h(\rightarrow \gamma \gamma)$ from gluon fusion at the HL-LHC and hl-100 tev hadron collider, Phys. Rev. D 100, 096001 (2019).

[69] M. Grazzini, G. Heinrich, S. Jones, S. Kallweit, M. Kerner, J. M. Lindert, and J. Mazzitelli, Higgs boson pair production at NNLO with top quark mass effects, J. High Energy Phys. 05 (2018) 059.
[70] K. Belotsky, D. Fargion, M. Khlopov, R. Konoplich, and K. Shibaev, Invisible Higgs boson decay into massive neutrinos of fourth generation, Phys. Rev. D 68, 054027 (2003).

[71] M. Carena, I. Low, N. R. Shah, and C. E. M. Wagner, Impersonating the standard model Higgs boson: Alignment without decoupling, J. High Energy Phys. 04 (2014) 015 .

[72] A. Celis, V. Ilisie, and A. Pich, LHC constraints on twoHiggs doublet models, J. High Energy Phys. 07 (2013) 053.

[73] J. Bernon, J. F. Gunion, H. E. Haber, Y. Jiang, and S. Kraml, Scrutinizing the alignment limit in two-Higgsdoublet models: $\mathrm{m}_{h}=125 \mathrm{GeV}$, Phys. Rev. D 92, 075004 (2015).

[74] S. Chang, S. K. Kang, J.-P. Lee, and J. Song, Higgs potential and hidden light Higgs scenario in two Higgs doublet models, Phys. Rev. D 92, 075023 (2015).

[75] D. Das and I. Saha, Search for a stable alignment limit in two-Higgs-doublet models, Phys. Rev. D 91, 095024 (2015).

[76] S. L. Glashow and S. Weinberg, Natural conservation laws for neutral currents, Phys. Rev. D 15, 1958 (1977).

[77] E. A. Paschos, Diagonal neutral currents, Phys. Rev. D 15, 1966 (1977).

[78] M. Aoki, S. Kanemura, K. Tsumura, and K. Yagyu, Models of Yukawa interaction in the two Higgs doublet model, and their collider phenomenology, Phys. Rev. D 80, 015017 (2009).

[79] A. Djouadi, The anatomy of electro-weak symmetry breaking. I: The Higgs boson in the standard model, Phys. Rep. 457, 1 (2008).

[80] R. Barbieri, A. Pomarol, R. Rattazzi, and A. Strumia, Electroweak symmetry breaking after LEP-1 and LEP-2, Nucl. Phys. B703, 127 (2004).

[81] G. Aad et al. (ATLAS Collaboration), Search for vector-like $B$ quarks in events with one isolated lepton, missing transverse momentum and jets at $\sqrt{s}=8 \mathrm{TeV}$ with the ATLAS detector, Phys. Rev. D 91, 112011 (2015).

[82] G. Aad et al. (ATLAS Collaboration), Search for production of vector-like quark pairs and of four top quarks in the lepton-plus-jets final state in $p p$ collisions at $\sqrt{s}=$ $8 \mathrm{TeV}$ with the ATLAS detector, J. High Energy Phys. 08 (2015) 105.

[83] G. Aad et al. (ATLAS Collaboration), Search for the production of single vector-like and excited quarks in the $W t$ final state in $p p$ collisions at $\sqrt{s}=8 \mathrm{TeV}$ with the ATLAS detector, J. High Energy Phys. 02 (2016) 110.

[84] G. Aad et al. (ATLAS Collaboration), Search for single production of vector-like quarks decaying into $\mathrm{Wb}$ in $\mathrm{pp}$ collisions at $\sqrt{s}=8 \mathrm{TeV}$ with the ATLAS detector, Eur. Phys. J. C 76, 442 (2016).

[85] G. Aad et al. (ATLAS Collaboration), Search for single production of a vector-like quark via a heavy gluon in the $4 b$ final state with the ATLAS detector in $p p$ collisions at $\sqrt{s}=8 \mathrm{TeV}$, Phys. Lett. B 758, 249 (2016).

[86] M. Aaboud et al. (ATLAS Collaboration), Search for pair production of vector-like top quarks in events with one lepton, jets, and missing transverse momentum in 
$\sqrt{s}=13 \mathrm{TeV} p p$ collisions with the ATLAS detector, J. High Energy Phys. 08 (2017) 052.

[87] M. Aaboud et al. (ATLAS Collaboration), Search for pair production of heavy vector-like quarks decaying to high$\mathrm{p}_{T} \mathrm{~W}$ bosons and $\mathrm{b}$ quarks in the lepton-plus-jets final state in pp collisions at $\sqrt{s}=13 \mathrm{TeV}$ with the ATLAS detector, J. High Energy Phys. 10 (2017) 141.

[88] M. Aaboud et al. (ATLAS Collaboration), Search for pair production of up-type vector-like quarks and for four-topquark events in final states with multiple $b$-jets with the ATLAS detector, J. High Energy Phys. 07 (2018) 089.

[89] M. Aaboud et al. (ATLAS Collaboration), Search for pair production of heavy vector-like quarks decaying into high- $p_{T} W$ bosons and top quarks in the leptonplus-jets final state in $p p$ collisions at $\sqrt{s}=13 \mathrm{TeV}$ with the ATLAS detector, J. High Energy Phys. 08 (2018) 048.

[90] M. Aaboud et al. (ATLAS Collaboration), Search for pair- and single-production of vector-like quarks in final states with at least one $Z$ boson decaying into a pair of electrons or muons in $p p$ collision data collected with the ATLAS detector at $\sqrt{s}=13 \mathrm{TeV}$, Phys. Rev. D 98, 112010 (2018).

[91] M. Aaboud et al. (ATLAS Collaboration), Search for pair production of heavy vector-like quarks decaying into hadronic final states in $p p$ collisions at $\sqrt{s}=13 \mathrm{TeV}$ with the ATLAS detector, Phys. Rev. D 98, 092005 (2018).

[92] M. Aaboud et al. (ATLAS Collaboration), Combination of the Searches for Pair-Produced Vector-Like Partners of the Third-Generation Quarks at $\sqrt{s}=13 \mathrm{TeV}$ with the ATLAS Detector, Phys. Rev. Lett. 121, 211801 (2018).

[93] S. Chatrchyan et al. (CMS Collaboration), Search for TopQuark Partners with Charge 5/3 in the Same-Sign Dilepton Final State, Phys. Rev. Lett. 112, 171801 (2014).

[94] V. Khachatryan et al. (CMS Collaboration), Search for pair-produced vectorlike B quarks in proton-proton collisions at $\sqrt{s}=8 \mathrm{TeV}$, Phys. Rev. D 93, 112009 (2016).

[95] V. Khachatryan et al. (CMS Collaboration), Search for vector-like charge $2 / 3 \mathrm{~T}$ quarks in proton-proton collisions at sqrt(s) = $8 \mathrm{TeV}$, Phys. Rev. D 93, 012003 (2016).

[96] V. Khachatryan et al. (CMS Collaboration), Search for single production of a heavy vector-like $\mathrm{T}$ quark decaying to a Higgs boson and a top quark with a lepton and jets in the final state, Phys. Lett. B 771, 80 (2017).

[97] A. M. Sirunyan et al. (CMS Collaboration), Search for electroweak production of a vector-like quark decaying to a top quark and a Higgs boson using boosted topologies in fully hadronic final states, J. High Energy Phys. 04 (2017) 136.

[98] A. M. Sirunyan et al. (CMS Collaboration), Search for single production of vector-like quarks decaying to a $\mathrm{Z}$ boson and a top or a bottom quark in proton-proton collisions at $\sqrt{s}=13 \mathrm{TeV}$, J. High Energy Phys. 05 (2017) 029.

[99] A. M. Sirunyan et al. (CMS Collaboration), Search for single production of vector-like quarks decaying into $\mathrm{a} b$ quark and a $\mathrm{W}$ boson in proton-proton collisions at $\sqrt{s}=13 \mathrm{TeV}$, Phys. Lett. B 772, 634 (2017).

[100] A. M. Sirunyan et al. (CMS Collaboration), Search for pair production of vector-like $\mathrm{T}$ and $\mathrm{B}$ quarks in single-lepton final states using boosted jet substructure in proton-proton collisions at $\sqrt{s}=13 \mathrm{TeV}$, J. High Energy Phys. 11 (2017) 085.

[101] A. M. Sirunyan et al. (CMS Collaboration), Search for single production of a vector-like $\mathrm{T}$ quark decaying to a $\mathrm{Z}$ boson and a top quark in proton-proton collisions at $\sqrt{s}=13 \mathrm{TeV}$, Phys. Lett. B 781, 574 (2018).

[102] A. Spiezia (CMS Collaboration), Search for single production of a vector-like T quark decaying to $\mathrm{tZ}$ with CMS at $\sqrt{s}=13 \mathrm{TeV}$, in 5th Large Hadron Collider Physics Conference (LHCP 2017) Shanghai, China (2017) [arXiv: 1708.03124].

[103] T. Han, H.E. Logan, B. McElrath, and L.-T. Wang, Phenomenology of the little Higgs model, Phys. Rev. D 67, 095004 (2003).

[104] M. Perelstein, M. E. Peskin, and A. Pierce, Top quarks and electroweak symmetry breaking in little Higgs models, Phys. Rev. D 69, 075002 (2004).

[105] G. Aad et al. (ATLAS Collaboration), Search for pair production of a new heavy quark that decays into a $W$ boson and a light quark in $p p$ collisions at $\sqrt{s}=8 \mathrm{TeV}$ with the ATLAS detector, Phys. Rev. D 92, 112007 (2015).

[106] A. M. Sirunyan et al. (CMS Collaboration), Search for long-lived particles using nonprompt jets and missing transverse momentum with proton-proton collisions at $\sqrt{s}=13$ TeV, Phys. Lett. B 797, 134876 (2019).

[107] R. D. Ball et al. (NNPDF Collaboration), Parton distributions for the LHC Run II, J. High Energy Phys. 04 (2015) 040 .

[108] M. Tanabashi et al. (Particle Data Group), Review of Particle Physics, Phys. Rev. D 98, 030001 (2018).

[109] S. Borowka, N. Greiner, G. Heinrich, S. P. Jones, M. Kerner, J. Schlenk, U. Schubert, and T. Zirke, Higgs Boson Pair Production in Gluon Fusion at Next-to-Leading Order with Full Top-Quark Mass Dependence, Phys. Rev. Lett. 117, 012001 (2016).

[110] S. Borowka, N. Greiner, G. Heinrich, S. P. Jones, M. Kerner, J. Schlenk, and T. Zirke, Full top quark mass dependence in Higgs boson pair production at NLO, J. High Energy Phys. 10 (2016) 107.

[111] J. Baglio, F. Campanario, S. Glaus, M. Mhlleitner, M. Spira, and J. Streicher, Gluon fusion into Higgs pairs at NLO QCD and the top mass scheme, Eur. Phys. J. C 79, 459 (2019).

[112] J. Baglio, F. Campanario, S. Glaus, M. M. Muhlleitner, J. Ronca, M. Spira et al., Higgs-pair production via gluon gusion at hadron colliders: NLO QCD corrections, arXiv:2003.03227.

[113] J. Amacker et al., Higgs self-coupling measurements using deep learning and jet substructure in the $b \bar{b} b \bar{b}$ final state, J. High Energy Phys. 12 (2020) 115.

[114] A. Adhikary, S. Banerjee, R. Kumar Barman, and B. Bhattacherjee, Resonant heavy Higgs searches at the HL-LHC, J. High Energy Phys. 09 (2019) 068.

[115] J. Amacker et al., Higgs self-coupling measurements using deep learning and jet substructure in the $b \bar{b} b \bar{b}$ final state, arXiv:2004.04240.

[116] S. Dawson et al., Working group report: Higgs boson, in Proceedings, 2013 Community Summer Study on the Future of U.S. Particle Physics: Snowmass on the 
Mississippi (CSS2013): Minneapolis, MN, USA (2013) [arXiv:1310.8361].

[117] M. Vos, Top physics beyond the LHC, in Proceedings, 9th International Workshop on Top Quark Physics (TOP 2016): Olomouc, Czech Republic (2016) [arXiv: 1701.06537].

[118] M. Vos, Review of top and EW physics at future colliders, Proc. Sci., EPS-HEP2017 (2017) 471.

[119] L.-B. Chen, H. T. Li, H.-S. Shao, and J. Wang, The gluon-fusion production of Higgs boson pair: $\mathrm{N}^{3} \mathrm{LO}$ QCD corrections and top-quark mass effects, J. High Energy Phys. 03 (2020) 072.

[120] S. Borowka, G. Heinrich, S. Jahn, S. P. Jones, M. Kerner, J. Schlenk, and T. Zirke, pySecDec: A toolbox for the numerical evaluation of multi-scale integrals, Comput. Phys. Commun. 222, 313 (2018).

[121] G. Heinrich, S. P. Jones, M. Kerner, G. Luisoni, and L. Scyboz, Probing the trilinear Higgs boson coupling in diHiggs production at NLO QCD including parton shower effects, J. High Energy Phys. 06 (2019) 066.

[122] D. Wardrope, E. Jansen, N. Konstantinidis, B. Cooper, R. Falla, and N. Norjoharuddeen, Non-resonant Higgs-pair production in the $b \bar{b} b \bar{b}$ final state at the LHC, Eur. Phys. J. C 75, 219 (2015).

[123] W. Yao, Studies of measuring Higgs self-coupling with $H H \rightarrow b \bar{b} \gamma \gamma$ at the future hadron colliders, in Proceedings, 2013 Community Summer Study on the Future of U.S. Particle Physics: Snowmass on the Mississippi (CSS2013): Minneapolis, MN, USA (2013) [arXiv:1308.6302].

[124] A. Papaefstathiou, L. L. Yang, and J. Zurita, Higgs boson pair production at the LHC in the $b \bar{b} W^{+} W^{-}$channel, Phys. Rev. D 87, 011301 (2013).

[125] J. H. Kim, K. Kong, K. T. Matchev, and M. Park, Probing the Triple Higgs Self-Interaction at the Large Hadron Collider, Phys. Rev. Lett. 122, 091801 (2019).

[126] G. Aad et al. (ATLAS Collaboration), Searches for Higgs boson pair production in the $h h \rightarrow b b \tau \tau$, $\gamma \gamma W W^{*}, \gamma \gamma b b, b b b b$ channels with the ATLAS detector, Phys. Rev. D 92, 092004 (2015).

[127] G. Aad et al. (ATLAS Collaboration), Search for Higgs boson pair production in the $b \bar{b} b \bar{b}$ final state from pp collisions at $\sqrt{s}=8 \mathrm{TeV}$ with the ATLAS detector, Eur. Phys. J. C 75, 412 (2015).

[128] M. Aaboud et al. (ATLAS Collaboration), Search for pair production of Higgs bosons in the $b \bar{b} b \bar{b}$ final state using proton-proton collisions at $\sqrt{s}=13 \mathrm{TeV}$ with the ATLAS detector, Phys. Rev. D 94, 052002 (2016).

[129] M. Aaboud et al. (ATLAS Collaboration), Search for pair production of Higgs bosons in the $b \bar{b} b \bar{b}$ final state using proton-proton collisions at $\sqrt{s}=13 \mathrm{TeV}$ with the ATLAS detector, J. High Energy Phys. 01 (2019) 030.

[130] A. M. Sirunyan et al. (CMS Collaboration), Search for a massive resonance decaying to a pair of Higgs bosons in the four b quark final state in protonproton collisions at $\sqrt{s}=13 \mathrm{TeV}$, Phys. Lett. B 781, 244 (2018).

[131] A. M. Sirunyan et al. (CMS Collaboration), Search for nonresonant Higgs boson pair production in the $\mathrm{b} \bar{b} \mathrm{~b} \bar{b}$ final state at $\sqrt{s}=13 \mathrm{TeV}$, J. High Energy Phys. 04 (2019) 112.
[132] G. Aad et al. (ATLAS Collaboration), Search for nonresonant Higgs boson pair production in the $b b \ell \nu \ell \nu$ final state with the ATLAS detector in $p p$ collisions at $\sqrt{s}=13 \mathrm{TeV}$, Phys. Lett. B 801, 135145 (2020).

[133] A. M. Sirunyan et al. (CMS Collaboration), Search for resonances decaying to a pair of Higgs bosons in the $b \bar{b} q \bar{q}^{\prime} \ell \nu$ final state in proton-proton collisions at $\sqrt{s}=13 \mathrm{TeV}$, J. High Energy Phys. 10 (2019) 125.

[134] M. Aaboud et al. (ATLAS Collaboration), Search for Resonant and Non-Resonant Higgs Boson Pair Production in the $b \bar{b} \tau^{+} \tau^{-}$Decay Channel in $p p$ Collisions at $\sqrt{s}=$ $13 \mathrm{TeV}$ with the ATLAS Detector, Phys. Rev. Lett. 121, 191801 (2018).

[135] G. Aad et al. (ATLAS Collaboration), Search For Higgs Boson Pair Production in the $\gamma \gamma b \bar{b}$ Final State using $p p$ Collision Data at $\sqrt{s}=8 \mathrm{TeV}$ from the ATLAS Detector, Phys. Rev. Lett. 114, 081802 (2015).

[136] M. Aaboud et al. (ATLAS Collaboration), Search for Higgs boson pair production in the $\gamma \gamma b \bar{b}$ final state with $13 \mathrm{TeV} p p$ collision data collected by the ATLAS experiment, J. High Energy Phys. 11 (2018) 040.

[137] A. M. Sirunyan et al. (CMS Collaboration), Search for Higgs boson pair production in the $\gamma \gamma \mathrm{b} \bar{b}$ final state in pp collisions at $\sqrt{s}=13 \mathrm{TeV}$, Phys. Lett. B 788, 7 (2019).

[138] M. Aaboud et al. (ATLAS Collaboration), Search for Higgs boson pair production in the $\gamma \gamma W W^{*}$ channel using $p p$ collision data recorded at $\sqrt{s}=13 \mathrm{TeV}$ with the ATLAS detector, Eur. Phys. J. C 78, 1007 (2018).

[139] M. Aaboud et al. (ATLAS Collaboration), Search for Higgs boson pair production in the $b \bar{b} W W^{*}$ decay mode at $\sqrt{s}=13 \mathrm{TeV}$ with the ATLAS detector, J. High Energy Phys. 04 (2019) 092.

[140] M. Aaboud et al. (ATLAS Collaboration), Search for Higgs boson pair production in the $W W^{(*)} W W^{(*)}$ decay channel using ATLAS data recorded at $\sqrt{s}=13 \mathrm{TeV}$, J. High Energy Phys. 05 (2019) 124.

[141] J. K. Behr, D. Bortoletto, J. A. Frost, N. P. Hartland, C. Issever, and J. Rojo, Boosting Higgs pair production in the $b \bar{b} b \bar{b}$ final state with multivariate techniques, Eur. Phys. J. C 76, 386 (2016).

[142] J. Alison et al., Higgs boson pair production at colliders: Status and perspectives, in Double Higgs Production at Colliders, edited by B. Di Micco, M. Gouzevitch, J. Mazzitelli, and C. Vernieri (2019).

[143] J. Alwall, M. Herquet, F. Maltoni, O. Mattelaer, and T. Stelzer, MadGraph 5: Going beyond, J. High Energy Phys. 06 (2011) 128.

[144] J. Alwall, R. Frederix, S. Frixione, V. Hirschi, F. Maltoni, O. Mattelaer, H.-S. Shao, T. Stelzer, P. Torrielli, and M. Zaro, The automated computation of tree-level and nextto-leading order differential cross sections, and their matching to parton shower simulations, J. High Energy Phys. 07 (2014) 079.

[145] T. Sjostrand, S. Ask, J. R. Christiansen, R. Corke, N. Desai, P. Ilten, S. Mrenna, S. Prestel, C. O. Rasmussen, and P.Z. Skands, An introduction to PYTHIA 8.2, Comput. Phys. Commun. 191, 159 (2015).

[146] J. de Favereau, C. Delaere, P. Demin, A. Giammanco, V. Lematre, A. Mertens, and M. Selvaggi (DELPHES 3 Collaboration), DELPHES 3, A modular framework for fast 
simulation of a generic collider experiment, J. High Energy Phys. 02 (2014) 057.

[147] M. Cacciari, G. P. Salam, and G. Soyez, The anti- $k_{t}$ jet clustering algorithm, J. High Energy Phys. 04 (2008) 063.

[148] M. Cacciari, G. P. Salam, and G. Soyez, FastJet user manual, Eur. Phys. J. C 72, 1896 (2012).

[149] ATLAS Collaboration, Study of the double Higgs production channel $H(\rightarrow b \bar{b}) H(\rightarrow \gamma \gamma)$ with the ATLAS experiment at the HL-LHC, CERN Tech. Report No. ATLPHYS-PUB-2017-001, 2017.
[150] ATLAS Collaboration, Measurement prospects of the pair production and self-coupling of the Higgs boson with the ATLAS experiment at the HL-LHC, CERN Tech. Report No. ATL-PHYS-PUB-2018-053, 2018.

[151] ATLAS Collaboration, Expected performance for an upgraded ATLAS detector at High-Luminosity LHC, CERN Tech. Report No. ATL-PHYS-PUB-2016-026, 2016.

[152] Expected performance of the ATLAS $b$-tagging algorithms in Run-2, CERN Tech. Report No. ATL-PHYS-PUB2015-022, 2015. 